\title{
A harmonic solution for two-dimensional adjoint QCD
}

\author{
Uwe Trittmann \\ Department of Physics, Otterbein University, Westerville, Ohio 43081, USA
}

(Received 12 June 2018; published 15 August 2018)

\begin{abstract}
Two-dimensional QCD with adjoint fermions has many attractive features, yet its single-particle content remains largely unknown. To lay the foundation for a crucially improved approximation of the theory's spectrum, we developed a method to find the basis of eigenstates using the symmetry structure of the asymptotic theory where pair production is disallowed. This method produces complete sets of multidimensional harmonic functions for the massless and the massive theory. Previously only part of such a basis was known. The method presented here should be applicable to other theories and has the promise of factoring out the long-range Coulomb-type part of interactions. The role of pair production and implications for the bosonized theory in the case of adjoint $\mathrm{QCD}_{2}$ are discussed.
\end{abstract}

DOI: 10.1103/PhysRevD.98.045011

\section{INTRODUCTION}

Two-dimensional adjoint quantum chromodynamics, $\mathrm{QCD}_{2 A}$, is a non-Abelian Yang-Mills theory coupled to fermions in the adjoint representation, and based on the Lagrangian

$$
\mathcal{L}=\operatorname{Tr}\left[-\frac{1}{4 g^{2}} F_{\mu \nu} F^{\mu \nu}+i \bar{\Psi} \gamma_{\mu} D^{\mu \Psi}\right]
$$

where $\Psi=2^{-1 / 4}\left(\begin{array}{l}\psi \\ \chi\end{array}\right)$, with $\psi$ and $\chi$ being $N \times N$ matrices. The field strength is $F_{\mu \nu}=\partial_{\mu} A_{\nu}-\partial_{\nu} A_{\mu}+i\left[A_{\mu}, A_{\nu}\right]$, and the covariant derivative is defined as $D_{\mu}=\partial_{\mu}+i\left[A_{\mu}, \cdot\right]$. Throughout the paper, light-cone coordinates $x^{ \pm}=\left(x^{0} \pm\right.$ $\left.x^{1}\right) / \sqrt{2}$ are used, where $x^{+}$plays the role of a time. We will work in the light-cone gauge, $A^{+}=0$, effectively omitting fermionic zero modes.

The theory is discussed extensively in the literature [1-6]. Why is $\mathrm{QCD}_{2 A}$ interesting? For starters, it is the simplest gauge theory apart from 't Hooft's model [7] that exhibits confinement. Additionally, $\mathrm{QCD}_{2 A}$ shows several features of higher-dimensional gauge theories. Its adjoint degrees of freedom (d.o.f.) mimic the transverse gluons which are dynamical obviously only in two or more spatial dimensions. Also, $\mathrm{QCD}_{2 A}$ exhibits a phase transition from confinement to screening in the limit of vanishing mass $m$ of the adjoint fermions. This mass furthermore parameterizes the supersymmetric behavior the theory exhibits at

Published by the American Physical Society under the terms of the Creative Commons Attribution 4.0 International license. Further distribution of this work must maintain attribution to the author(s) and the published article's title, journal citation, and DOI. Funded by SCOAP ${ }^{3}$. $m^{2}=g^{2} N$. The theory has an exponentially increasing density of bound states according to [1,3]. Since the density behaves like $\rho(M) \sim e^{M / T_{H}}$ for large bound state masses $M$, one expects a deconfinement transition at the Hagedorn temperature $T_{H}$. Also, this transition depends crucially on the fermion mass. In particular, the Hagedorn temperature goes to zero in the limit $m \rightarrow 0$, driven by the vanishing string tension at the deconfinement transition. There are several unresolved issues, for instance the existence of Regge-trajectories of single-particle (bound) states. Few single-particle states have been identified to date $[3,4,8]$, which has led to speculations that there is no Regge trajectory in massless $\mathrm{QCD}_{2 A}$ [6]. This is understandable in a screening theory in which long strings should fall apart, but leaves one to explain what happens to the many singletrace states. It seems that most of them have to be interpreted as multiparticle states $[3,8]$. But since some of them can be projected out by bosonization [9] and the rest is close to threshold in a formulation of the theory with a finite discretization parameter, this must be considered an unsettled question. On the other hand, the theory is the simplest available ${ }^{1}$ exhibiting these interesting features, so the hope is to learn something about the mechanisms underlying more realistic gauge theories. Indeed, there recently has been a renewed interest in three- and fourdimensional versions of the theory $[10,11]$.

While the ultimate goal is a solution of the full theory, our aim here is much more modest. As a foundation for future work, we construct in Sec. II a complete set of eigenstates for the asymptotic theory based on symmetries which are broken in the full theory, thereby expanding work begun in [12]. The basis is a set of linear combinations of multidimensional harmonic functions subject to

\footnotetext{
${ }^{1}$ It is a low-dimensional, manifestly finite theory.
} 
the symmetry constraints furnished by the light-cone Hamiltonian derived from Eq. (1). Since the asymptotic theory has disjoint sectors with different parton numbers, the basis states can be organized by parton number $r$, which results in $r-1$ excitation numbers responsible for the multidimensionality of the solution. Technically, the basis will be appropriate only in the high excitation number limit. Empirically, we find that even the lowest states are well represented, probably due to their large separation in (bound state) mass. In the full theory, particle pair production couples the disjoint parton sectors, and eigenfunctions of the full theory will be linear combinations of basis states of different parton numbers. Thus, the harmonic basis states can be used to discuss the role of particle creation and annihilation, as envisioned in [1]. This is done in Sec. III B. As applications of our method we briefly describe a program to solve the full theory with numerical methods in Sec. III A, and point out implications for the bosonized version of the theory in Sec. III C. Finally, we discuss the results and general applicability of the method in Sec. IV.

\section{CONSTRUCTING A HARMONIC BASIS}

\section{A. Introductory remarks}

Starting from the $\mathrm{QCD}_{2 A}$ Lagrangian, Eq. (1), the dynamics of a system of adjoint fermions interacting via a nondynamical gluon field in two dimensions can be described by a light-cone momentum operator $P^{+}$and Hamiltonian operator $P^{-}$. The two operators are expressed in terms of fermionic operators subject to the anticommutation relation

$$
\left\{b_{i j}\left(k^{+}\right), b_{l k}^{\dagger}\left(p^{+}\right)\right\}=\delta\left(k^{+}-p^{+}\right)\left(\delta_{i l} \delta_{j k}-\frac{1}{N} \delta_{i j} \delta_{k l}\right) .
$$

To wit

$$
P^{+}=\int_{0}^{\infty} d k k b_{i j}^{\dagger}(k) b_{i j}(k),
$$

with

$$
\begin{aligned}
A\left(k_{i}\right) & =\frac{1}{\left(k_{4}-k_{2}\right)^{2}}-\frac{1}{\left(k_{1}+k_{2}\right)^{2}}, \\
B\left(k_{i}\right) & =\frac{1}{\left(k_{2}+k_{3}\right)^{2}}-\frac{1}{\left(k_{1}+k_{2}\right)^{2}}, \\
C(k) & =\int_{0}^{k} d p \frac{k}{(p-k)^{2}}, \\
D\left(k_{i}\right) & =\frac{1}{\left(k_{1}-k_{4}\right)^{2}}-\frac{1}{\left(k_{2}-k_{4}\right)^{2}},
\end{aligned}
$$

where the trace-splitting term $D\left(k_{i}\right)$ can be omitted at large $N_{c}$, and the trace-joining term is proportional to $B\left(k_{i}\right)$. The structure of the Hamiltonian $P^{-}$displayed in Eq. (4) is

$P^{-}=P_{m}^{-}+P_{\text {ren }}^{-}+P_{P C, s}^{-}+P_{P C, n s}^{-}+P_{P V}^{-}+P_{\text {finite } N}^{-}$.
The mass term $P_{m}^{-}$is dropped in the massless theory, but the renormalization operator $P_{\text {ren }}^{-}$needs to be included. Partonnumber violating terms, $P_{P V}^{-}$, couple blocks of different parton number. Parton-number conserving interactions $P_{P C}^{-}$ are block diagonal, and may include $\operatorname{singular}(s)$ or nonsingular $(n s)$ functions of the parton momenta. The finite $N$ term $P_{\text {finite } N}^{-}$is proportional to $D\left(k_{i}\right)$. For details see $[2,3,12]$.

The problem is cast into an eigenvalue equation

$$
2 P^{+} P^{-}|\Psi\rangle \equiv H_{\mathrm{LC}}|\Psi\rangle=M^{2}|\Phi\rangle .
$$

Namely, the light-cone Hamiltonian $H_{\mathrm{LC}}$ acts on an eigenket $|\Psi\rangle$ yielding the mass (squared) of a bound state as the eigenvalue. The eigenkets are in general linear combinations of states of definite parton(fermion) number $r$ 


$$
\left|\Phi_{r}\right\rangle=\int_{0}^{\frac{1}{r}} d x_{1}\left(\prod_{i=2}^{r-1} \int_{x_{1}}^{1-(r-1) x_{1}-\sum_{j=2}^{i-1} x_{j}} d x_{i}\right) \frac{\phi_{r}\left(x_{1}, x_{2}, \ldots, x_{r}\right)}{N_{c}^{r / 2}} \operatorname{Tr}\left[b\left(-x_{1}\right) \cdots b\left(-x_{r}\right)\right]|0\rangle .
$$

The wave functions $\phi_{r}$ distribute momentum between the partons. Note that there are only $r-1$ integrations since total momentum can be set to unity. The integration is over the effective Hilbert space, which looks complicated due to elimination of redundant operator combinations like $\operatorname{Tr}[b(x) b(y) b(z)]=\operatorname{Tr}[b(y) b(z) b(x)]$. The explicit shape of the integration domain is displayed to emphasize that for $r>3$ the wave function cannot be reconstructed ${ }^{2}$ in the region around the middle of the naive Hilbert space $[0,1]^{r-1}$.

In the asymptotic limit, where only highly excited states are considered, both parton-violation and mass terms can be neglected. As a consequence the asymptotic theory splits into decoupled sectors with fixed parton numbers subject to 't Hooft-like equations

$$
\frac{M^{2}}{g^{2} N} \phi_{r}\left(x_{1}, \ldots, x_{r}\right)=-\sum_{i=1}^{r}(-1)^{(r+1)(i+1)} \int_{-\infty}^{\infty} \frac{\phi_{r}\left(y, x_{i}+x_{i+1}-y, x_{i+2}, \ldots, x_{i+r-1}\right)}{\left(x_{i}-y\right)^{2}} d y .
$$

The total momentum is set to unity, and thus the momentum fractions $x_{i}$ add up to one, $\sum_{i} x_{i}=1$. Clearly, the number of partons $r$ is even (odd) for bosonic (fermionic) states. In $[1,12]$, additionally the approximation

$$
\int_{0}^{1} \frac{d y}{(x-y)^{2}} \phi(y) \approx \int_{-\infty}^{\infty} \frac{d y}{(x-y)^{2}} \phi(y)
$$

was used. Mathematically this is helpful because the solutions of the eigenvalue problem then are harmonic functions, see Eq. (15). The approximation makes sense physically, because for the highly excited states the integral is dominated by the interval around $x=y$ which is associated with the long-range Coulomb-type force.

In [12], we showed that the integral equation (12) can be solved algebraically using the ansatz

$$
\left|n_{1}, n_{2}, \ldots n_{r-1}\right\rangle \doteq \prod_{j}^{r-1} e^{i \pi n_{j} x_{j}}=\phi_{r}\left(x_{1}, x_{2}, \ldots, x_{r}\right),
$$

where $x_{r}=1-\sum_{j}^{r-1} x_{j}$. The ansatz is motivated by its simplest $(r=2)$ version, which solves the 't Hooft equation of fundamental $\mathrm{QCD}_{2}$ [7]

$$
\frac{M^{2}}{g^{2} N} e^{i \pi n x}=-\int_{-\infty}^{\infty} \frac{d y}{(x-y)^{2}} e^{i \pi n y}=\pi|n| e^{i \pi n x},
$$

where the excitation number $n$ is integer. We thus use the single-particle states of a Hamiltonian appropriate for the problem to construct a Fock basis, inspired by [13].

Since the integral equation (12) is more involved than the 't Hooft equation, we have to symmetrize the ansatz (14) to comply with the constraints inherent in the Hamiltonian (10). Namely, the solutions of the adjoint 't Hooft problem have to be (pseudo)cyclic,

$$
\phi_{r}\left(x_{1}, x_{2}, \ldots, x_{r}\right)=(-1)^{r+1} \phi_{r}\left(x_{2}, x_{3} \ldots, x_{r}, x_{1}\right),
$$

since the fermions are real. To implement this constraint we introduce the cyclic permutation operator

$$
\mathcal{C}:\left(x_{1}, x_{2}, \ldots, x_{r}\right) \rightarrow\left(x_{2}, x_{3}, \ldots, x_{r}, x_{1}\right) .
$$

Since the Hamiltonian is unchanged by a color index reversal of its operators, the string or trace of fermionic operators in the states (11) can be reversed at will. The solutions can therefore be organized into sectors of definite parity under the orientation symmetry,

$$
\mathcal{T}: b_{i j} \rightarrow b_{j i}
$$

The two symmetry operators act on the ansatz, Eq. (14), as follows.

$$
\begin{aligned}
\mathcal{C}:\left|n_{1}, n_{2}, \ldots, n_{r-1}\right\rangle \rightarrow(-1)^{n_{r-1}}\left|-n_{r-1}, n_{1}-n_{r-1}, n_{2}-n_{r-1}, \ldots, n_{r-2}-n_{r-1}\right\rangle, \\
\mathcal{T}:\left|n_{1}, n_{2}, \ldots, n_{r-1}\right\rangle \rightarrow(-1)^{n_{1}}\left|-n_{1}, n_{r-1}-n_{1}, n_{r-2}-n_{1}, \ldots, n_{2}-n_{1}\right\rangle .
\end{aligned}
$$

In [12], we constructed eigenfunctions in the two- and three-parton sectors $(r=2,3)$ for both the massive and the massless theory, with bound-state masses

$$
M^{2}=g^{2} N \pi\left(\left|n_{1}\right|+\left|n_{r-1}\right|+\sum_{k=1}^{r-2}\left|n_{k}-n_{k+1}\right|\right) .
$$

\footnotetext{
${ }^{2}$ Meaning we cannot determine the wave function in this region from symmetries and its values close to the domain boundaries. For $r=2$ we can: knowing $\phi_{2}(x)$ in $\left[0, \frac{1}{2}\right]$ and $\phi_{2}(x)=-\phi_{2}(1-x)$ is obviously enough.
} 
The crucial ingredient of the method is the symmetrization due to $\mathcal{C}$,

$$
\left|n_{1}, n_{2}, \ldots n_{r-1}\right\rangle_{\mathrm{sym}} \equiv \frac{1}{\sqrt{r}} \sum_{k=1}^{r}(-1)^{(r-1)(k-1)} \mathcal{C}^{k-1}\left|n_{1}, n_{2}, \ldots n_{r-1}\right\rangle,
$$

where $\mathcal{C}^{0}=1$, because only the symmetrized states reflect the (pseudo)cyclic structure of the Hamiltonian.

While the $r=2,3$ wave functions reproduce known (DLCQ) solutions of the theory remarkably well down to the lowest states, the ansatz fails to work for $r>3$ in general. In [12], we were able to express a subset of the $r=4$ solutions as a linear combination of the symmetrized four-parton states. These solutions coincide with the ones derived earlier [1]. To introduce notation and show the complexity of the problem, we display a five-parton state symmetrized under $\mathcal{C}$ and $\mathcal{T}$, i.e., of definite $C$ and $T$ quantum numbers. ${ }^{3}$ Note that the state consists of $4 r=20$ statelets, characterized by an $(r-1)$-tuple of (ordered) excitation numbers $n_{i}$ [here: $\left.(n, m, l, k)\right]$

$$
\begin{aligned}
\mid \phi_{5}, n, & \left.m, l, k ; \bar{M}^{2}=|n|+|n-m|+|m-l|+|l-k|+|k|\right\rangle_{T} \\
= & |n, m, l, k\rangle+(-1)^{k}|-k, n-k, m-k, l-k\rangle+(-1)^{l}|k-l,-l, n-l, m-l\rangle \\
& +(-1)^{m}|l-m, k-m,-m, n-m\rangle+(-1)^{n}|m-n, l-n, k-n,-n\rangle \\
& +T\left[(-1)^{n}|-n, k-n, l-n, m-n\rangle+|k, l, m, n\rangle+(-1)^{k}|l-k, m-k, n-k,-k\rangle\right. \\
& \left.+(-1)^{l}|m-l, n-l,-l, k-l\rangle+(-1)^{m}|n-m,-m, k-m, l-m\rangle\right] .
\end{aligned}
$$

Typically, these states are paired with a partner state of negative excitation numbers to create a real wave function, i.e., a sine or cosine. It is convenient to do so with an additional symmetry in mind, which we'll introduce in the next section.

\section{B. Exhaustive symmetrization}

As is well known, the eigenfunctions of the massive theory have to vanish when one parton momentum is zero to guarantee Hermiticity of the Hamiltonian [7]

$$
\phi_{n}\left(0, x_{2}, \ldots, x_{n}\right)=0
$$

This is sometimes called a boundary condition because it behaves as one, which is misleading-after all, we are trying to solve an integral and not a differential equation. In the massive two-parton sector (or the simpler fundamental theory $\mathrm{QCD}_{2 f}$ [7]), this leads to sine eigenfunctions, and to cosine eigenfunctions in the massless theory. Clearly, the general verdict is that the Hilbert space of the theory splits into two disjoint sets of functions: one even and the other one odd. It seems that the consequences of this straightforward observation have not been fully realized. This is not surprising, since the answer to the simple question-under which transformation or symmetry the eigenfunctions are odd or even-is trivial for few partons, and fairly complicated for many. Indeed, the symmetry in question is

\footnotetext{
${ }^{3}$ While $T$ is an ordinary quantum number reflecting a symmetry of the Hamiltonian, $C$ (or rather the set of $C_{i}$ ) is fixed by the constraint that the state has an (anti)cyclic wave function, Eq. (16), as required by the structure of the Hamiltonian as a sum over permutations of the parton momenta.
}

manifest only in the parton sectors with $r \geq 4$, because it is redundant with $\mathcal{C}$ and $\mathcal{T}$ otherwise. To get a handle on it, note that while physically it is true that we need to manage the behavior of the wave functions at the boundaries of the domain of integration, mathematically boundary conditions are not the right tool. Rather, we have to implement symmetries that will result in the desired behavior of the wave functions at the boundary-just like the $\mathcal{C}$ symmetrization assures that the state so constructed is an eigenstate of the Hamiltonian. We alluded to such a symmetry in [12], but the general method is more involved.

For massive fermions, we need the wave functions to vanish on the hyperplanes characterized by $x_{i}=0$ for at least one $x_{i}$. Since our wave function ansatz (14) is modular, we can simply add for every term of the form $e^{i \pi \sum_{j} n_{j} x_{j}}$ another term with opposite sign that is the same for $x_{i}=0$ but different ${ }^{4}$ for $x_{i} \neq 0$. This idea can be realized by introducing $r-1$ symmetry operators $\mathcal{S}_{i}$ which we might call lower-dimensional inversions, because they invert all but one of the excitation numbers

$$
\begin{aligned}
\mathcal{S}_{i}: & \left|n_{1}, n_{2}, \ldots, n_{i} \ldots, n_{r-1}\right\rangle \\
& \rightarrow\left|-n_{1},-n_{2}, \ldots, n_{i}-n_{i+1}-n_{i-1}\left(1-\delta_{1 i}\right), \ldots,-n_{r-1}\right\rangle .
\end{aligned}
$$

The replacement of the $i$ th excitation number is such that the mass (squared) of the state remains invariant, see Eq. (19). This symmetry is hidden in the three-parton sector $r=3$ because the low-dimensional inversion can be

\footnotetext{
${ }^{4}$ Because the wave function would otherwise be identically zero, of course.
} 
TABLE I. Characteristics of the lowest states in the first few parton sectors of the asymptotic theory including their quantum numbers $T I S$. The sectors are labeled with a subscript indicating behavior under $\mathcal{T}\left({ }_{ \pm}\right)$and superscripts signifying massless $\left({ }^{0}\right)$ and massive fermions $\left(^{\mu}\right)$. For an explanation of the discrepancy between the $T$ quantum number and the $\mathcal{T}$ sector, see Appendix B.

\begin{tabular}{|c|c|c|c|c|}
\hline$r$ & T I S & Sector & Excitation numbers of lowest states & Masses $\left(g^{2} N \pi\right)$ \\
\hline \multirow[t]{2}{*}{2} & -+ & $|o\rangle_{+}^{0}$ & $(1),(3),(5),(7)$ & $1,3,5,7$ \\
\hline & -- & $|e\rangle_{+}^{\mu}$ & $(2),(4),(6),(8)$ & $2,4,6,8$ \\
\hline \multirow[t]{4}{*}{3} & ++ & $|e e\rangle_{-}^{0}$ & $(0,0),(2,2),(4,2),(4,0),(6,2)$ & $0,4,8,8,12$ \\
\hline & -- & $|e e\rangle_{+}^{0}$ & $(4,2),(6,2),(8,2),(8,4)$ & $8,12,16,16$ \\
\hline & +- & $|e e\rangle_{-}^{\mu}$ & $(2,0),(4,0),(6,2),(6,0)$ & $4,8,12,12$ \\
\hline & -+ & $|e e\rangle_{+}^{\mu}$ & $(6,2),(8,2),(10,4),(10,2)$ & $12,16,20,20$ \\
\hline \multirow[t]{4}{*}{4} & +-- & $|o e o\rangle_{+}^{0}$ & $(1,2,3),(3,-2,-1),(3,-2,-3),(5,-2,-1)$ & $6,10,12,14$ \\
\hline & -++ & $|o e o\rangle_{-}^{0}$ & $(1,0,1),(1,-2,-1),(3,0,1),(2,-2,-1)$ & $4,6,8,10$ \\
\hline & +-+ & $|e e e\rangle_{+}^{\mu}$ & $(4,-2,0),(6,-2,0),(6,10,10),(8,10,6),(8,10,10)$ & $12,16,20,20,20$ \\
\hline & -+- & $|e e e\rangle_{-}^{\mu}$ & $(4,0,2),(4,-2,0),(6,4,6),(6,-2,0)$ & $12,12,16,16$ \\
\hline \multirow[t]{4}{*}{5} & +++ & $|e e e e\rangle_{+}^{0}$ & $(0,0,0,0),(2,2,2,2),(2,4,4,4),(4,4,4,2),(4,4,4,4)$ & $0,4,8,8,8$ \\
\hline & --- & $|e e e e\rangle_{-}^{0}$ & $(4,4,4,2),(4,6,6,6),(4,6,4,2),(6,6,4,2),(4,8,8,8)$ & $8,12,12,12,16$ \\
\hline & ++- & $|e e e e\rangle_{+}^{\mu}$ & $(4,6,6,4),(6,8,8,6),(6,10,10,6),(8,10,10,6)$ & $12,16,20,20$ \\
\hline & --+ & $|e e e\rangle_{-}^{\mu}$ & $(8,10,10,6),(8,12,10,6),(8,14,12,8)$ & $20,24,28$ \\
\hline \multirow[t]{4}{*}{6} & -++ & $\mid$ oeoeo $\rangle_{+}^{0}$ & $(1,2,3,2,1),(1,2,3,4,3),(5,4,3,2,1),(3,4,5,4,3)$ & $6,8,10,10$ \\
\hline & +-- & $\mid$ oeoeo $\rangle_{-}^{0}$ & $(\ldots)$ & \\
\hline & ++- & $|e е e е e\rangle_{-}^{\mu}$ & & \\
\hline & --+ & $|e е e е e\rangle_{+}^{\mu}$ & & \\
\hline
\end{tabular}

expressed in terms of the other symmetry operations ${ }^{5}$ ( $\mathcal{S}=\mathcal{T C}^{2}$, see Eq. (26) of [12]), so that $r=4$ is the lowest parton sector in which the full symmetry unfolds.

In Sec. II D, we will see that additional $\mathcal{S}$ operators creep in, so let's call the set of lower-dimensional inversions $\mathcal{E}=\left\{\mathcal{S}_{1}, \mathcal{S}_{2}, \ldots, \mathcal{S}_{N(r)}\right\}$. For a given parton number $r$, the maximal number $N(r)$ of independent $\mathcal{S}$ operators can be determined from a group theoretical argument. In practice, the argument boils down to combinatorics: the number of operators of the permutation group of order $r$ with inversion is $2 r$ !. We derive the full symmetry group $\mathcal{G}$ in Sec. II D and use only its generic properties here. The group choice is intuitive as we will see, since in the $r$ parton sector, we are in essence permuting $r$ objects. That the objects (the partons) carry different momenta is irrelevant here. We are thus symmetrizing the wave functions under the set of $N(r) \mathcal{S}$ symmetries. Later we will see that there is only one common multiplicative $Z_{2}$ quantum number $S$. Tentatively, we write

$$
|r\rangle_{\text {sym }}=\left(1+\sum_{i}^{N(r)} S_{i} \mathcal{S}_{i=1}\right)|r\rangle_{\mathrm{sym}}
$$

where $|r\rangle_{\text {sym }}$ is the ansatz state $\left|n_{1}, n_{2}, \ldots, n_{r-1}\right\rangle$ symmetrized under the cyclic group $\langle\mathcal{C}\rangle$, Eq. (20).

To include all symmetries in our approach, it is convenient to make the inversion of excitation numbers explicit with the operator

$$
\mathcal{I}:\left|n_{1}, n_{2}, \ldots, n_{r-1}\right\rangle \rightarrow\left|-n_{1},-n_{2}, \ldots,-n_{r-1}\right\rangle .
$$

\footnotetext{
${ }^{5}$ At $r=2$ the situation is fully degenerate with $\mathcal{C}=\mathcal{T}=\mathcal{S}$ up to signs.
}

Clearly, $\mathcal{I}$ is a $Z_{2}$ operator, and states even and odd under $\mathcal{I}$ simply represent cosine and sine wave functions, respectively. We can then write down an orthonormal set of basis states in all sectors of the theory, characterized by their $Z_{2}$ quantum numbers $(T, I, S)$ under the symmetry transformations $\mathcal{T}, \mathcal{I}$, and $\mathcal{S}$, and their excitation numbers $n_{i}$ collected in $|r\rangle$

$$
\begin{aligned}
|r\rangle_{\text {FullSym }} \equiv & \mathcal{G}|r\rangle=\left\{\frac{(1+T \mathcal{T})(1+I \mathcal{I})}{\sqrt{2 r ! \mathcal{N}}}\right. \\
& \left.\times \sum_{k=0}^{r-1}(-)^{(r-1) k} \mathcal{C}^{k}\left(1+\sum_{i}^{N(r)} S_{i} \mathcal{S}_{i=1}\right)|r\rangle\right\},
\end{aligned}
$$

where $\mathcal{N}$ is the volume of the Hilbert space in the $r$ parton sector,

$$
\mathcal{N}=\int_{0}^{\frac{1}{r}} d x_{1}\left(\prod_{i=2}^{r-1} \int_{x_{1}}^{1-(r-1) x_{1}-\sum_{j=2}^{i-1} x_{j}} d x_{i}\right)=\frac{1}{r !},
$$

cf. Appendix of Ref. [12]. The excitation numbers can, in general, be even or odd integers. Since the states, Eq. (25), are by construction eigenstates of the light-cone Hamiltonian with masses given by Eq. (19), they furnish, in principle, a full solution ${ }^{6}$ of asymptotic $\mathrm{QCD}_{2 A}$-keeping in mind that we made the approximation, Eq. (13).

\footnotetext{
${ }^{6}$ This construction should also settle the issue raised by 't Hooft in the footnote of his seminal paper [7]: since the "boundary conditions" are really symmetrizations, they must hold order by order in $N_{c}$.
} 


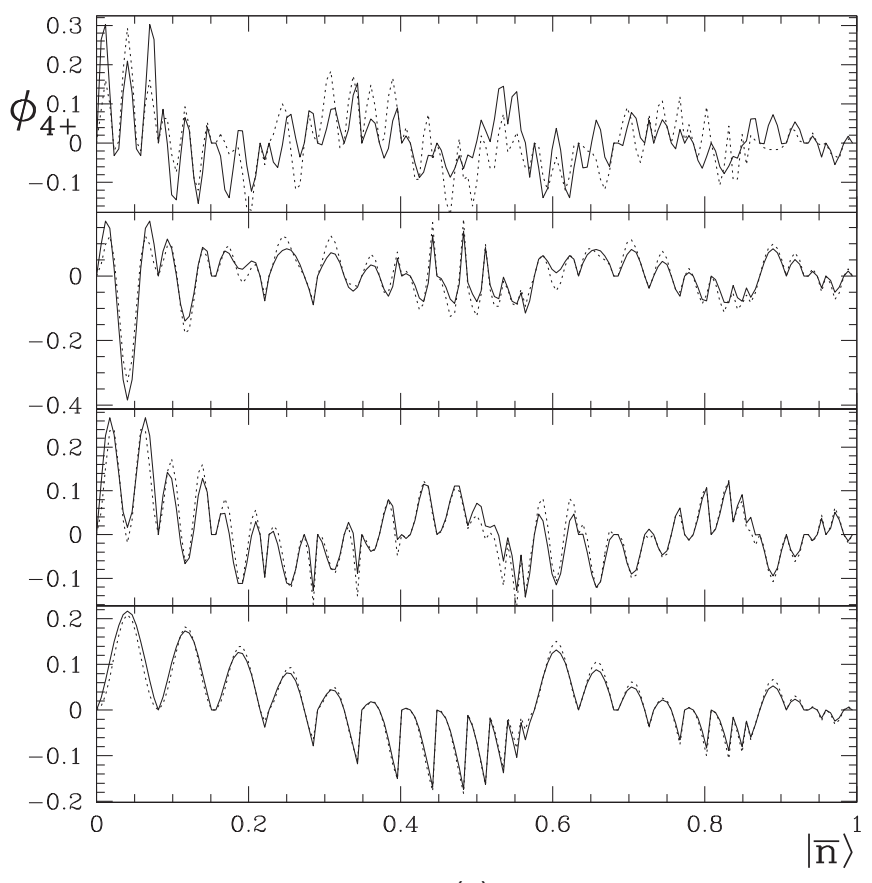

(a)

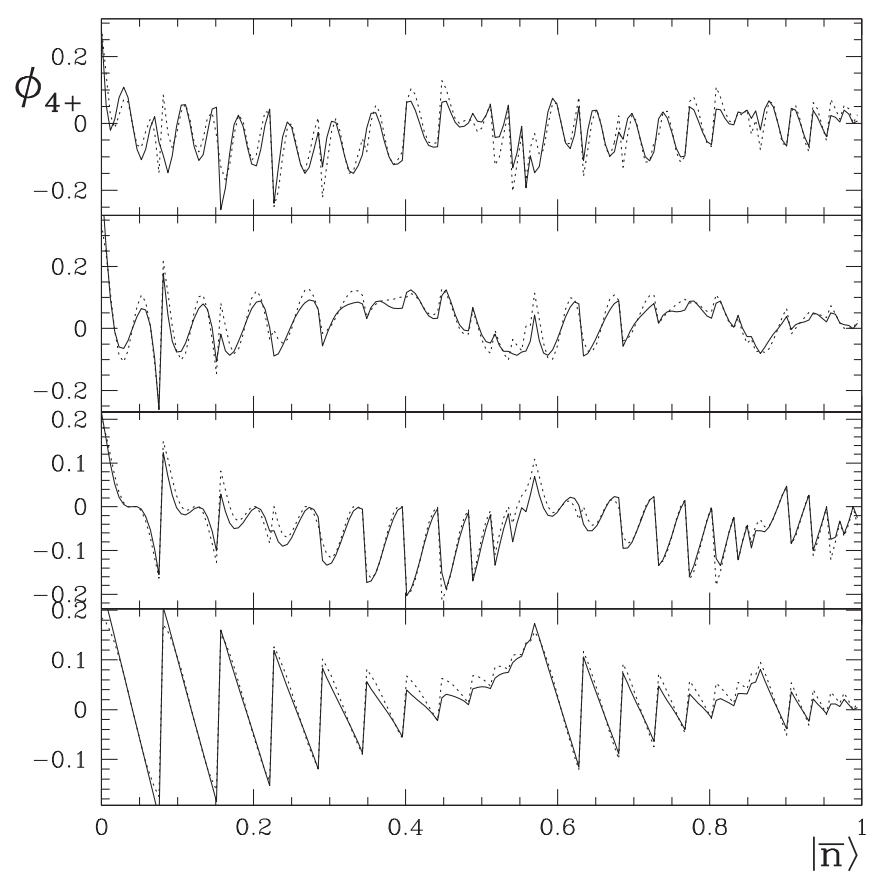

(b)

FIG. 1. Lowest DLCQ (solid lines, $K=32$ ) and asymptotic eigenfunctions (dashed lines) in the massless four-parton sectors, i.e., $\mid$ oeo $\rangle$ states with $\mu \equiv m^{2} / g^{2} N=0$, plotted as a function of the normalized basis state number $\bar{n}$. (a) $T+$, hence $I-S-$. (b) $T-$, hence $I+S+$. We used a rather low harmonic resolution $K$ to increase readability, since we do not need to worry about convergence here.

\section{Classification of states}

In practice, most sectors prescribed by Eq. (25) are empty. As can be gleaned from the symmetry operations, Eqs. (18), (23), and (24), only two sets of quantum numbers lead to viable states. Namely, only states with all even excitation numbers, and states with alternating odd and even numbers give rise to bona fide states. In the latter case, the first and last excitation numbers must be odd, so that these states only exist in the even parton sectors. Even so, most of these excitation number combinations are not viable due to cancellations of terms. For example, bona fide states are only found in the $(T \pm, I \mp)$ four-parton sectors and in the $(T \pm, I \pm)$ five-parton sectors, see Table I. In the odd parton sectors, these four sectors (for both $S \pm$ ) are indeed the four sectors necessary to describe the two $\mathcal{T}$-sectors of the massive and the massless theory, respectively. For an even number of partons, the addition of the mixed excitation number states ( $\mid$ odd, even, odd $\rangle \equiv$ $|o e o\rangle$ for $r=4$ ) does, of course, not lead to more sectors. Rather, two even excitation number sectors do not yield bona fide states. For an explanation, see Appendix A. For instance, in the four parton sector, only $(T \pm, I \mp, S \pm)$ in the even excitation number sector and $(T \pm, I \mp, S \mp)$ with mixed even and odd $n_{i}$ are viable; the former are the two sectors of the massive theory, and the latter represent the two massless sectors. This is a straightforward generalization from the earlier findings [12] that in the two-parton sectors cosines with odd excitation number represent the massless and sines with even excitation number the massive theory, whereas all excitation numbers in the three-parton sector are even.

To check our musings, we make contact with known results. Our solution (25) reproduces the results of Ref. [1] Eq. (4.13) and classifies them as $|e e e ; T-I+S-\rangle$ states. The wave functions (4.13) of [1] exhibit only two (not three) excitation numbers, because they represent a subset of the full set of wave functions. We thus find that there are more states than anticipated, and that the counting of states is more involved; it does not seem to lend itself to a stringmotivated parametrization. Note that the solution (4.13) in [1] is much more compact and looks differently (double sines versus triple cosines), but this is a superficial disagreement and the price one has to pay for generality: 12 terms $^{7}$ of (4.13) in [1] vs. 48 terms in Eq. (25) at $r=4$. Next we check how accurate our algebraic solution is by comparing to a numerical (DLCQ) calculation, see Figs. 1 and 2. Some masses are degenerate at $r=4$ as opposed to $r<4$, and the agreement is not as good as in the threeparton sector [12], due to considerable mixing of states of equal mass. Recall that the algebraic eigensolutions were derived by making the approximation Eq. (13); using the correct limits of the integral evidently induces interactions between the algebraic basis states. As can be gleaned from Figs. 1 and 2, the discrepancy between algebraic and

\footnotetext{
${ }^{7}$ Obviously, a sine has two exponential terms.
} 


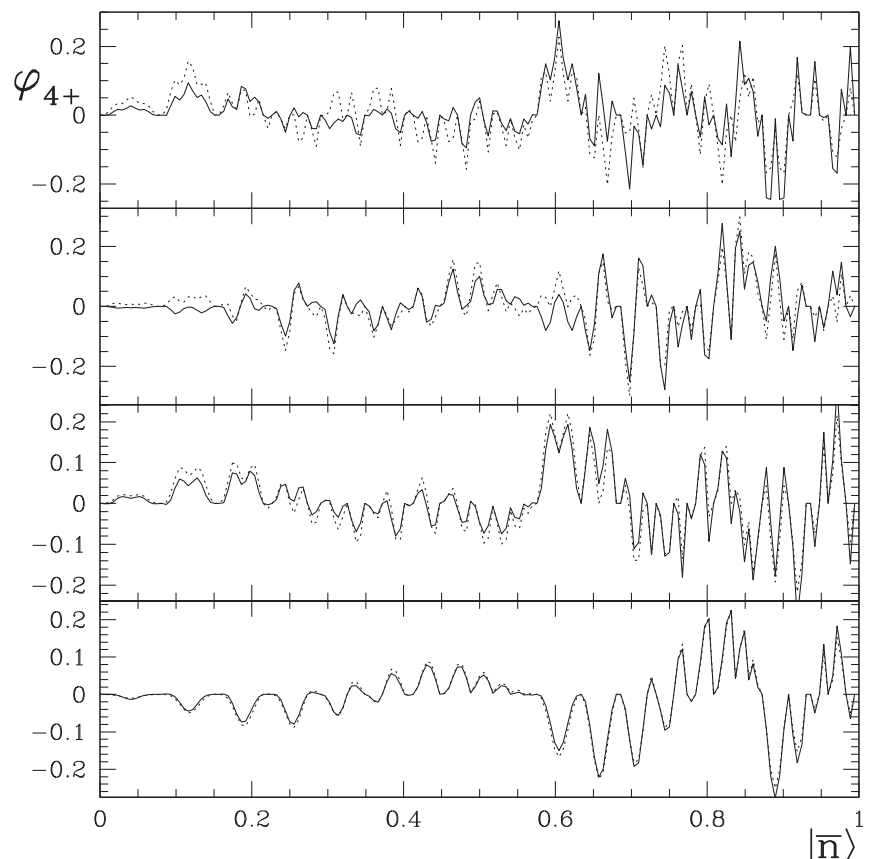

(a)

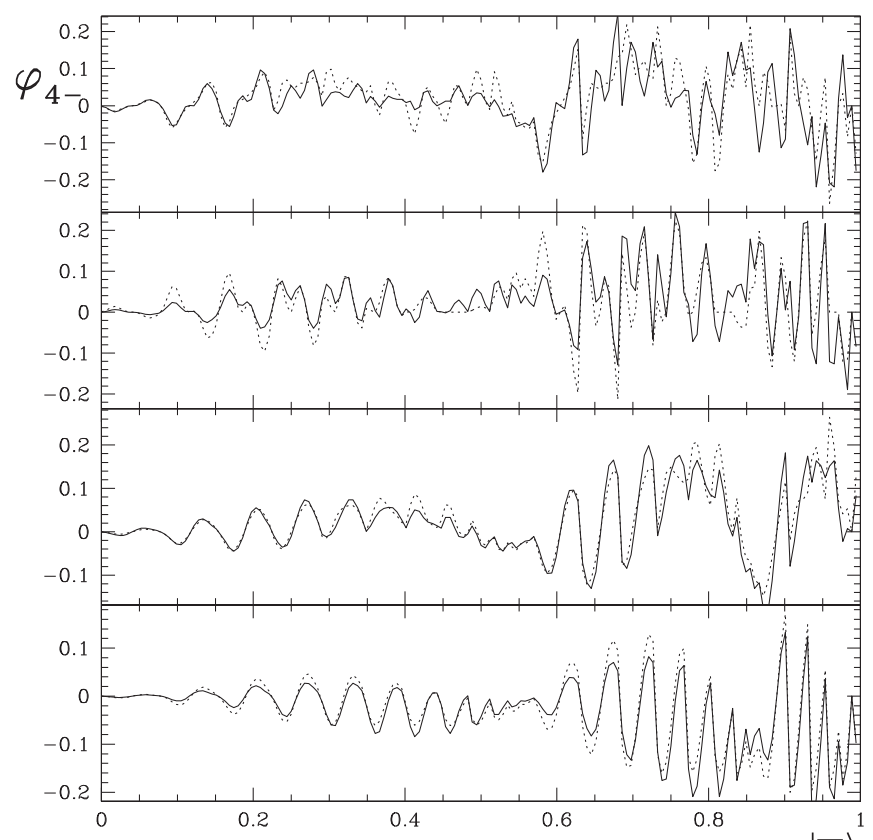

(b)

FIG. 2. Lowest eigenfunctions of the massive theory in the four-parton sectors. Solid lines represent numerical results as emulated in DLCQ by letting $\mu=4$, dashed lines the algebraic eigenfunctions. Note that for $\mu=1$, the theory is supersymmetric. (a) $\mathcal{T}$ even eigenfunctions. (b) $\mathcal{T}$ odd eigenfunctions.

numerical solutions is noticeable even in the massless sector, where the masses are not degenerate. This heralds the worsening of our approximation at large parton number $r$. Namely, the effective volume of the Hilbert space decreases as $1 / r$ !, see Eq. (26). As a consequence, the integral looks less and less like $\left(\int_{-\infty}^{\infty} d x\right)^{r-1}$, although the core motivation for the approximation (that the region around the singularity is the most important) remains valid.

What do the exact wave functions tell us? First off, the massless and massive sectors show significant differences.
The massive sector is more straightforward, perhaps due to the more stringent constraint of wave functions vanishing on the $x_{i}=0$ hyperplanes. Even the lowest states exhibit degenerate masses in the massive, but not the massless theory. Also, the bound-state masses are much lower in the massless sector. This is more surprising than it sounds, since we are omitting the mass term in the asymptotic limit. The difference in bound-state mass is thus generated by symmetry alone. The lowest four states are in the massive theory $(\mu \neq 0)$

$$
\begin{array}{rlrl}
|1\rangle_{+-+}^{\mu \neq 0} & =|4,-2,0\rangle_{12}, & |1\rangle_{-+-}^{\mu \neq 0}=|4,0,2\rangle_{12}, \\
|2\rangle_{+-+}^{\mu \neq 0}=|6,-2,0\rangle_{16}, & |2\rangle_{-+-}^{\mu \neq 0}=|4,-2,0\rangle_{12}, \\
|3\rangle_{+-+}^{\mu \neq 0}=\frac{1}{\sqrt{2}}\left(|6,10,10\rangle_{20}+|8,10,6\rangle_{20}\right), & |3\rangle_{-+-}^{\mu \neq 0}=|6,4,6\rangle_{16}, \\
|4\rangle_{+-+}^{\mu \neq 0}=|8,10,10\rangle_{20}, & |4\rangle_{-+-}^{\mu \neq 0}=|6,-2,0\rangle_{16} .
\end{array}
$$

In the massless theory, they look like

$$
\begin{array}{ll}
|1\rangle_{+--}^{\mu=0}=|1,2,3\rangle_{6}, & |1\rangle_{-++}^{\mu=0}=|1,0,1\rangle_{4}, \\
|2\rangle_{+--}^{\mu=0}=|3,-2,-1\rangle_{10}, & |2\rangle_{-++}^{\mu=0}=|1,-2,-1\rangle_{6}, \\
|3\rangle_{+--}^{\mu=0}=|3,-2,-3\rangle_{12}, & |3\rangle_{-++}^{\mu=0}=|3,0,1\rangle_{8}, \\
|4\rangle_{+--}^{\mu=0}=|5,-2,-1\rangle_{14}, & |4\rangle_{-++}^{\mu=0}=|3,-2,-1\rangle_{10},
\end{array}
$$


where the indices represent the TIS quantum numbers on the right-hand states, and the mass (squared in units $\left.g^{2} N \pi\right)$ on the left-hand states. Note that the excitation numbers $n_{i}$ are not unique. For instance, at $r=4$ each state has up to $2 r !=48$ different tuples. One way of classifying the state is to pick one statelet's numbers to represent the entire state, e.g., by choosing the lowest positive number for $n_{i}$ followed by the smallest absolutes $\left|n_{i}\right|$ as in Eq. (28).

\section{Some group theory}

The solutions (25) are right cosets of the subgroup $\mathcal{B}$ of transformations associated with the full domain of integration (the "bulk"). Namely, $\mathcal{B}$ is the direct product of inversions $\mathcal{I}$, reorientations $\mathcal{T}$ and cyclic permutations $\mathcal{C}$

$\mathcal{B}=\left\{1, \mathcal{C}, \mathcal{C}^{2}, \ldots \mathcal{C}^{r-1}, \mathcal{T}, \mathcal{T} C, \ldots \mathcal{T} C^{r-1}, \mathcal{I}, \mathcal{I} C \ldots, \mathcal{I} \mathcal{T} C^{r-1}\right\}$.

We can construct a partition of the full group $\mathcal{G}$ of symmetry transformations of $\mathrm{QCD}_{2 A}$ by acting on all elements of $\mathcal{B}$ with the lower-dimensional inversions $\mathcal{S}$ concerning symmetrization on the hyperplanes forming the boundary of the integration domain collected in the $\operatorname{set}^{8} \mathcal{E}$ alluded to in Sec. II B

$$
\mathcal{E}=\left\{\mathcal{S}_{1}, \mathcal{S}_{2}, \ldots \mathcal{S}_{1 / 2(r-1) !-1}\right\}
$$

In general, we find $N(r)=\frac{1}{2}(r-1) !-1$ independent lower-dimensional inversions. We work with the right cosets here, because we want to make explicit the symmetrization of the state under the cyclic subgroup $\langle\mathcal{C}\rangle$, as required by the structure of the Hamiltonian, as implicitly defined in Eq. (12). Since the order of $\mathcal{B}$ is $|\mathcal{B}|=2_{\mathcal{T}} \times 2_{\mathcal{I}} \times r_{\mathcal{C}}$ and its operators act on all elements of $\mathcal{E}$ plus the identity, a fully symmetrized state contains $2 r$ ! "statelets" owing to the $2 r$ ! independent automorphisms that can be formed on a set of $r$ objects (here: momentum fractions) if inversions are allowed.

The general theme is hard to prove, so we simply checked with a computer algorithm that the order of $\mathcal{G}$ is indeed $|\mathcal{G}|=2 r$ !一in particular, that it is finite. This is expected because permutations form subgroups of the symmetric group. From a physical point of view, permutations are reorderings which leave the boundstate masses invariant, and thus symmetries of the Hamiltonian.

To check the order of $\mathcal{G}$ we proceeded as follows. By acting on all $4 r$ statelets $\mathcal{B}_{i}|r\rangle$ with the first operator $\mathcal{S} \equiv \mathcal{S}_{1}$ we produced the left coset $\mathcal{S}_{1} \mathcal{B}$. To keep the

\footnotetext{
${ }^{8}$ We refer to it as the exhaustive set $\mathcal{E}$, because its elements are exhausting the "symmetrization space." It is not a group, because it is not closed under composition of its members which may produce elements of $\mathcal{B}$.
}

TABLE II. The $\mathcal{E}$ part of the group multiplication table in the five-parton sector organized as left coset elements. Since there is a small number of primary operators, the table has been transposed, in the sense that the column-heading operators act on the row-leading operators, e.g., $\mathcal{S}_{1} \mathcal{S}_{2}=\mathcal{S}_{6}$. It is obvious that $\mathcal{E}$ is not a subgroup of $\mathcal{G}$; multiplying $\mathcal{S}$ operators generates $\mathcal{B}$ operators.

\begin{tabular}{|c|c|c|c|c|c|}
\hline & $\mathcal{S}_{1}$ & $\mathcal{S}_{2}$ & $\mathcal{S}_{3}$ & $\mathcal{S}_{4}$ & $\mathcal{S}_{5}$ \\
\hline$S_{1}$ & id & $\mathcal{S}_{11} \mathcal{I} \mathcal{T}$ & $\mathcal{S}_{7}$ & $\mathcal{S}_{11} \mathcal{C}^{3}$ & $\mathcal{S}_{7} \mathcal{I} \mathcal{T}$ \\
\hline$S_{2}$ & $\mathcal{S}_{6}$ & id & $\mathcal{S}_{10}$ & $\mathcal{S}_{6} \mathcal{I} \mathcal{T} \mathcal{C}^{3}$ & $\mathcal{S}_{10} \mathcal{I} \mathcal{T} \mathcal{C}$ \\
\hline $\mathcal{S}_{3}$ & $\mathcal{S}_{7}$ & $\mathcal{S}_{8}$ & id & $\mathcal{S}_{7} \mathcal{I} \mathcal{T} \mathcal{C}^{4}$ & $\mathcal{S}_{8} \mathcal{I} \mathcal{T C}$ \\
\hline $\mathcal{S}_{4}$ & $\mathcal{S}_{11} \mathcal{C}^{3}$ & $\mathcal{S}_{6} \mathcal{I} \mathcal{T} \mathcal{C}^{3}$ & $\mathcal{S}_{11} \mathcal{I} \mathcal{T C}$ & id & $\mathcal{S}_{6} \mathcal{C}^{4}$ \\
\hline $\mathcal{S}_{5}$ & $\mathcal{S}_{8} \mathcal{C}^{4}$ & $\mathcal{S}_{10} \mathcal{I} \mathcal{T} \mathcal{C}$ & $\mathcal{S}_{8} \mathcal{I} \mathcal{T C}$ & $\mathcal{S}_{10} \mathcal{C}$ & id \\
\hline $\mathcal{S}_{6}$ & $\mathcal{S}_{2}$ & $\mathcal{S}_{4} \mathcal{I} \mathcal{T} \mathcal{C}^{3}$ & $\mathcal{S}_{9} \mathcal{I} \mathcal{T} \mathcal{C}^{2}$ & $\mathcal{S}_{2} \mathcal{I} \mathcal{T} \mathcal{C}^{3}$ & $\mathcal{S}_{4} \mathrm{C}$ \\
\hline $\mathcal{S}_{7}$ & $\mathcal{S}_{3}$ & $\mathcal{S}_{9}$ & $\mathcal{S}_{1}$ & $\mathcal{S}_{3} \mathcal{I} \mathcal{T} \mathcal{C}^{4}$ & $\mathcal{S}_{1} \mathcal{I} \mathcal{T}$ \\
\hline $\mathcal{S}_{8}$ & $\mathcal{S}_{5} \mathcal{C}$ & $\mathcal{S}_{3}$ & $\mathcal{S}_{5} \mathcal{I} \mathcal{T} \mathcal{C}$ & $\mathcal{S}_{9} \mathcal{I} \mathcal{I} \mathcal{C}^{4}$ & $\mathcal{S}_{3} \mathcal{I} \mathcal{T C}$ \\
\hline $\mathcal{S}_{9}$ & $\mathcal{S}_{10} \mathcal{I} \mathcal{I} \mathcal{C}^{2}$ & $\mathcal{S}_{7}$ & $\mathcal{S}_{6} \mathcal{I} \mathcal{I} \mathcal{C}^{2}$ & $\mathcal{S}_{8} \mathcal{I} \mathcal{T} \mathcal{C}^{4}$ & $\mathcal{S}_{11}$ \\
\hline$S_{10}$ & $\mathcal{S}_{0} \mathcal{I} \mathcal{T} \mathcal{C}^{2}$ & $\mathcal{S}_{5} \mathcal{I} \mathcal{T C}$ & $\mathcal{S}_{2}$ & $\mathcal{S}_{5} \mathcal{C}^{4}$ & $\mathcal{S}_{2} \mathcal{I} \mathcal{T C}$ \\
\hline 10 & $\mathcal{S}_{4} \mathcal{C}^{2}$ & $\mathcal{S}_{1} \mathcal{I} \mathcal{T}$ & $\mathcal{S}_{4} \mathcal{I} \mathcal{T} \mathcal{C}$ & $\mathcal{S}_{1} \mathcal{C}^{2}$ & $\mathcal{S}_{9}$ \\
\hline
\end{tabular}

symmetry of the Hamiltonian manifest, we need however the right coset $\mathcal{B S}_{1}$. Recall that terms of odd $\mathcal{C}$-parity must be negative in the even $r$ sectors to ensure the alternating signs of the cyclic permutations in the Hamiltonian Eq. (12). Since in general the cosets are not normal, $\mathcal{S}_{1} \mathcal{B} \neq \mathcal{B} \mathcal{S}_{1}$, we do not off-hand know which $\mathcal{C}$-parity the members of a left coset of $\mathcal{B}$ might have. In general, both the left and the right coset will therefore contain new operators, which we'll have to include in our growing set of operators to move towards group closure. We must then act with these new operators on $\mathcal{B}$ as well as on the existing right cosets, which in turn will yield new operators, right and left cosets. This process continues until no new operators arise-if the group is finite. It is thus a nontrivial test of our hypothesis. We find that the algorithm always converges on the expected number of operators or statelets, namely $2 r !=240,1440,10080$, 80640 for $r=5,6,7,8$ and beyond.

To construct an actual eigenfunction, we need to solve one more problem. It is the assignment of $S$ parity to the individual statelets, in the sense that some of them will carry an odd power of $S$ quantum number, and some an even power. Recall that $S^{2}=1$ since $\mathcal{S}$ is a $Z_{2}$ operation. It is convenient to introduce the notion of a primary operator. Of the $\frac{1}{2}(r-1) !-1 \mathcal{S}$ operators in the $r$ parton sector, naively only $r-1$ (associated with the $r-1$ excitation numbers) seem necessary to ensure that the wave function is vanishing (maximal) on the hyperplanes $x_{i}=0$ in the massive (massless) theory, respectively. We call those $r-1$ operators together with an additional operator ${ }^{9} \mathcal{S}_{r}$ the

\footnotetext{
${ }^{9}$ While not intuitive, the last operator is necessary to complete the map $\mathcal{S}_{i} \times\langle\mathcal{C}\rangle \rightarrow\langle\mathcal{C}\rangle \mathcal{S}_{i}$, since the cyclic subgroup $\langle\mathcal{C}\rangle$ is of order $r$, not $r-1$.
} 


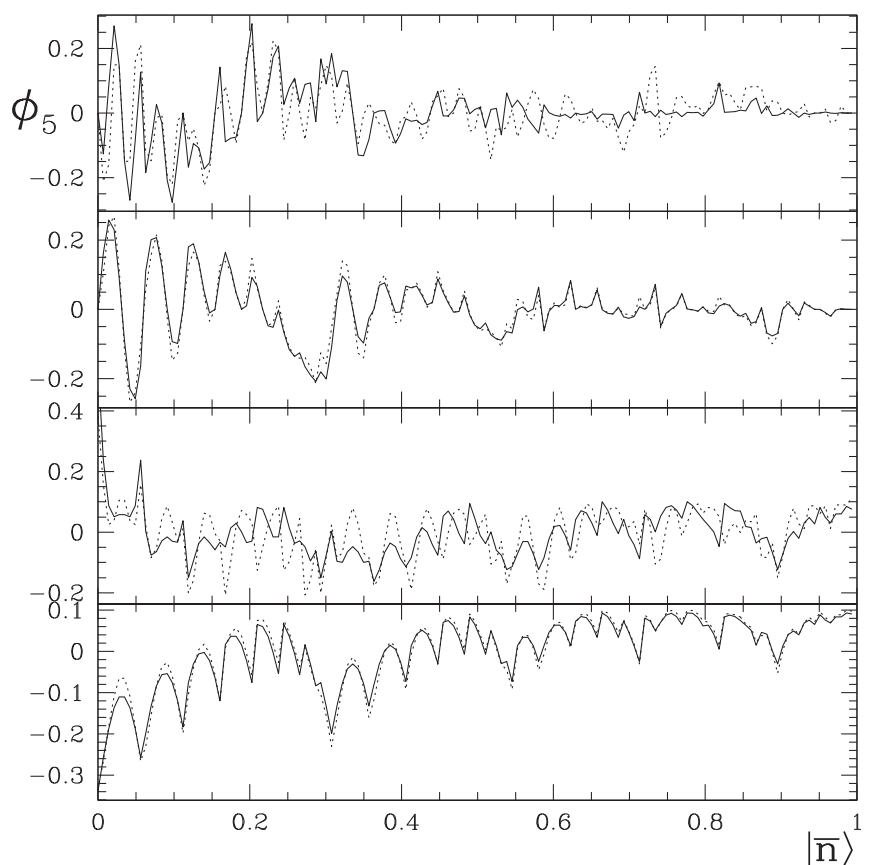

(a)

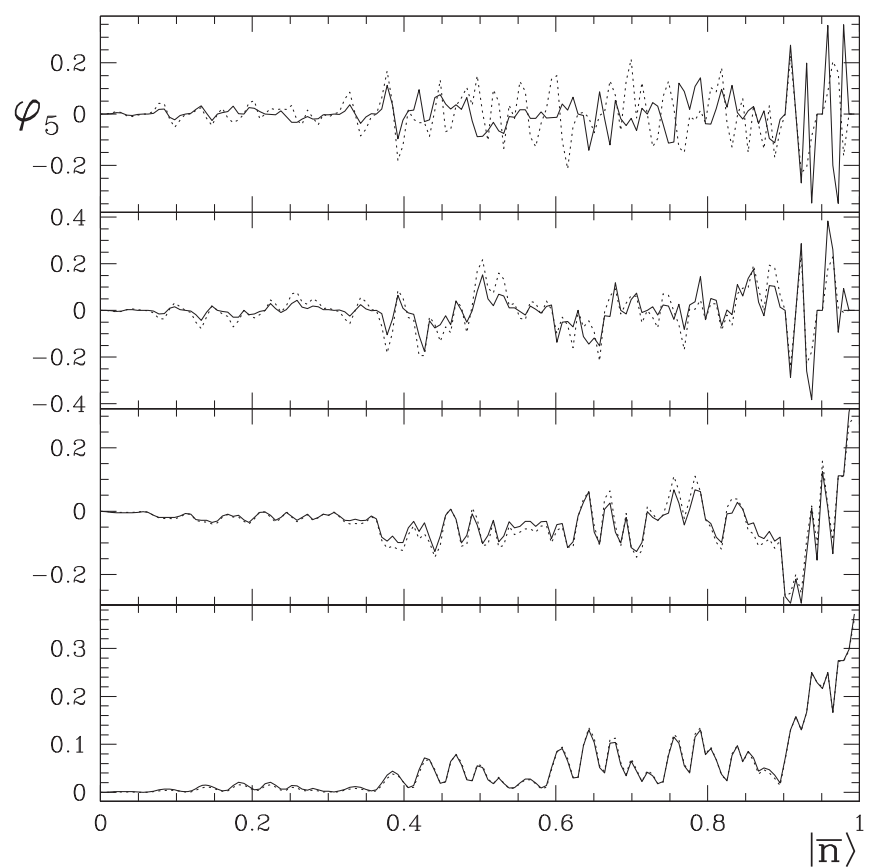

(b)

FIG. 3. Lowest eigenfunctions in all five-parton sectors. Solid lines represent numerical (DLCQ) results, dashed lines the algebraic eigenfunctions. (a) Massless theory $(\mu=0)$. (b) Massive theory $(\mu=4)$. The lowest two eigenfunctions are $\mathcal{T}$ even, the upper two $\mathcal{T}$ odd.

primary operators. They form a conjugacy class under $\langle\mathcal{C}\rangle$ and have similar properties, since ${ }^{10}$

$$
\mathcal{S}_{i}=\mathcal{C}^{i-1} \mathcal{S C}^{r-i+1}
$$

It is not hard to show that the primary operators fulfill the following pseudocommutation relations

$$
\mathcal{C}^{j} \mathcal{S}_{i}=\mathcal{S}_{\bmod _{r}^{\prime}(i+j)} \mathcal{C}^{j} \text { and }\left(\mathcal{T} \mathcal{C}^{j}\right) \mathcal{S}_{i}=\mathcal{S}_{\bmod _{r}^{\prime}(r-i+j+1)}\left(\mathcal{T} \mathcal{C}^{j}\right)
$$

where $\bmod _{r}^{\prime}$ is congruence modulo $r$ shifted by one, so that $\mathcal{S}_{0}=\mathcal{S}_{r}$ and $\mathcal{S}_{r+1}=\mathcal{S}_{1}$. In other words, the $\mathcal{S}$ operators commute with the members of the subgroup $\mathcal{B}$ under loss of their identity: they transmute into a different primary operator. But then we are done! These relations allow us to compute all other operators in the symmetry group $\mathcal{G}$. In fact, we must keep adding operators until the $2 r$ ! slots in the full symmetry group are exhausted. The product of two primary operators can be evaluated as follows due to the associativity group axiom ${ }^{11}$

$$
\begin{aligned}
\mathcal{S}_{k}\left(\mathcal{C}^{j} \mathcal{S}_{i}\right) & =\left(\mathcal{S}_{k} \mathcal{C}^{j}\right) \mathcal{S}_{i}=\mathcal{C}^{j}\left(\mathcal{S}_{\bmod _{r}^{\prime} k-j} \mathcal{S}_{i}\right) \\
& =\left(\mathcal{S}_{k} \mathcal{S}_{\bmod _{r}^{\prime} i+j}\right) \mathcal{C}^{j} \quad \text { for } 1 \leq i, j, k \leq r .
\end{aligned}
$$

\footnotetext{
${ }^{10}$ This relation is not unique, for instance $\mathcal{S}_{r-1}=(\mathcal{T C}) \mathcal{S}(\mathcal{T C})$ also.

${ }^{11}$ The $\mathcal{T}$ version of this relation is $\mathcal{S}_{k}\left(\mathcal{T} \mathcal{C}^{j} \mathcal{S}_{i}\right)=$ $\mathcal{T} \mathcal{C}^{j}\left(\mathcal{S}_{\bmod _{r}^{\prime}(r-k+j+1)} \mathcal{S}_{i}\right)=\left(\mathcal{S}_{k} \mathcal{S}_{\bmod _{r}^{\prime}(r-i+j+1)}\right) \mathcal{T} \mathcal{C}^{j}$.
}

The second half is the commutation relation for the secondary operator $\mathcal{S}_{\bmod _{r}^{\prime}(k-j)} \mathcal{S}_{i}$. As an example, consider $r=5$. First one shows with Eqs. (31)-(33) that the operator compositions $\mathcal{S}_{1} \mathcal{S}_{2}, \mathcal{S}_{1} \mathcal{S}_{3}$, and $\mathcal{S}_{2} \mathcal{S}_{3}$ cannot be written in terms of powers of $\mathcal{C}, \mathcal{T}, \mathcal{I}$, or $\mathcal{S}_{i}(i=1, \ldots 5)$. In this sense, these three combinations are "new" operators, and relabeled $\mathcal{S}_{6}, \mathcal{S}_{7}$, and $\mathcal{S}_{8}$, respectively. One then iterates by acting with existing operators on these operators, and puts any further "new" operators into $\mathcal{G}$. Often though, an equivalent operator combination will already exist in $\mathcal{G}$. For example, when acting with $\mathcal{S}_{2}$ on the right-coset statelet $\mathcal{C} S_{3}|r=5\rangle$ one finds using Eq. (33)

$$
\mathcal{S}_{2}\left(\mathcal{C S}_{3}\right)|r=5\rangle=\mathcal{C S}_{1} \mathcal{S}_{3}|r=5\rangle=\mathcal{C S}_{7}|r=5\rangle,
$$

and no new operator needs to be added. Finding new operators thus essentially reduces to the word problem of abstract algebra. Proceding along this way, a group multiplication table can be computed. We display part of it for $r=5$ in Table II. As a corollary we note that these identities show that the Hamiltonian symmetrization constraint (fixed signs under $\langle\mathcal{C}\rangle$ ) is intact. Namely, the $\mathcal{T}^{i} \mathcal{C}^{j}$ component of a right coset of $\mathcal{B}$ of any $\mathcal{S}_{k}$ (whether primary or not) is mapped onto the $\mathcal{T}^{i} \mathcal{C}^{j}$ component of a right coset of some other operator $\mathcal{S}_{k^{\prime}}$.

So what are the relative signs of the individual terms? The signs within the $\mathcal{B}$-blocks ( $4 r$ statelets of the right cosets $\mathcal{B} S_{i}$ ) are fixed by the Hamiltonian and the $T$ and $I$ quantum numbers. At $r=4$ the assignment of the $S$ quantum number is simple if counter-intuitive: both the $\mathcal{S}_{1}$ and the $\mathcal{S}_{2}$ block 




(a)

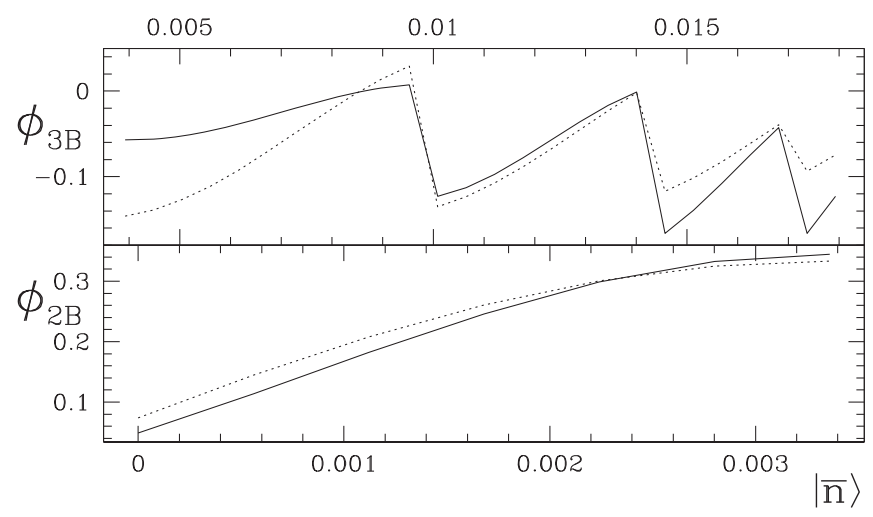

(b)

FIG. 4. (a) Massless six-parton $\mathcal{T}$ even eigenfunctions of asymptotic $\mathrm{QCD}_{2 A}$ at $K=20$. (b) The two- and three-parton parts of the lowest $\mathcal{T}$ even eigenfunction of the bosonized theory at $K_{B}=14$ which is equivalent to $K=28$ in the fermionic theory. In both graphs, DLCQ eigenfunctions are plotted with solid lines and "eLCQ" or model wave functions with dashed lines.

carry an additional $S$ sign (32 statelets); only the identity block's eight statelets do not acquire a sign. This seems unbalanced, but works due to sign-cancellations unique to the four-parton sector. At $r=5$ the orders of group and subgroups are such that we get an even split into two "halfgroups." Namely, the one quarternary and four secondary operators plus the identity are $S$ even and the primary operators plus a peculiar secondary operator ${ }^{12} \mathcal{S}_{9}$ are odd and carry an $S$ sign. This makes $r !=120$ statelets with an $S$ sign, and 120 without. For an algorithm that is more universally applicable, see Appendix A.

This completes our construction of the most general solution in all parton sectors. As a cross-check we construct the eigenfunctions in the five-parton sector and compare them to numerical solutions in Fig. 3. Figure 4(a) shows the comparison for some six-parton eigenfunctions. We used the rather low harmonic resolutions $K=23$ and $K=20$, because otherwise the plots get too "crowded"; agreement is just as good at higher $K$ of course. As we glean from Fig. 3, the agreement is near-perfect for the lowest $\mathcal{T}$ even state in the massive theory, good in the massive theory in general, and fair in the massless theory. This might be due to the fact that the density of states is much higher in the massless theory. Apparently, the condition that the wave function vanish for zero parton momenta is quite restrictive. Given that the number of statelets rises to 1440 at $r=6$ the agreement between algebraic eigenfunctions and numerical solution seems surprisingly good in Fig. 4(a).

It is indeed quite remarkable how perfectly the features of the theory are represented by the properties of the harmonic basis. It is tempting to speculate that this approach-which one might call "exhaustively-symmetrized light-cone quantization (eLCQ)"- -applies to other theories, at least in two dimensions where light-cone coordinates are the natural

\footnotetext{
${ }^{12}$ It commutes with the $\mathcal{B}$ subgroup, i.e., $\mathcal{B} S_{9}=S_{9} \mathcal{B}$, which does not imply commutation of individual subgroup elements $\mathcal{B}_{i}$ with $\mathcal{S}_{9}: \mathcal{B}_{i} S_{9} \neq S_{9} \mathcal{B}_{i}$ in general.
}

language. Hopefully, the treatment of the present toy model is a harbinger of wider applicability.

\section{APPLICATIONS}

\section{A. Using “eLCQ": Some suggestions}

Since we have found a complete set of basis states for the asymptotic theory in Sec. II, we can systematically approximate the full theory in a basis-function approach. This should be pretty straightforward, but implementing the algorithm in the higher parton-sectors is too tedious to be presented here, so we only sketch the general idea. Namely, we can use the set of coupled integral equations derived in [3] from the Hamiltonian Eq. (4) for the full wave functions $f_{r}$ in the $r$-parton sectors. The full equation couples sectors of different parton number; $f_{r}$ and $f_{r \pm 2}$ appear in the equation.

We expand the full eigenfunctions $f_{r}\left(x_{1}, x_{2}, \ldots, x_{r}\right)$ into a complete set of asymptotic eigenfunctions $\phi_{r, \vec{n}}$

$$
f_{r}\left(x_{1}, x_{2}, \ldots, x_{r}\right)=\sum_{\vec{n}} c_{r, \vec{n}} \phi_{r, \vec{n}}\left(x_{1}, x_{2}, \ldots, x_{r}\right),
$$

where $\vec{n}$ represents a tuple of $r-1$ excitation numbers. Formally, the integral equation looks like

$$
\begin{aligned}
M^{2} f_{r} & =M^{2} \sum_{\vec{n}} c_{r, \vec{n}} \phi_{r, \vec{n}}\left(x_{1}, x_{2}, \ldots, x_{r}\right) \\
& =2 P^{+} P^{-} \sum_{\vec{n}} c_{r, \vec{n}} \phi_{r, \vec{n}}\left(x_{1}, x_{2}, \ldots, x_{r}\right) .
\end{aligned}
$$

If we project onto the asymptotic eigenfunctions characterized by $(s, \vec{m})$, we get an equation for the associated coefficient

$$
\begin{aligned}
& M^{2} \int d^{r} x f_{s, \vec{m}}^{*}(\vec{x}) f_{r}(\vec{x})=M^{2} \sum_{r, \vec{n}} \int d^{r} x c_{r, \vec{n}} f_{s, \vec{m}}^{*} f_{r, \vec{n}} \\
& =M^{2} \sum_{r, \vec{n}} c_{r, \vec{n}} \delta_{s, r} \delta_{\vec{n}, \vec{m}}=M^{2} c_{s, \vec{m}},
\end{aligned}
$$


where $\vec{x}$ represents the $r$ momentum fractions $x_{i}$, and $\int d^{r} x$ the integration over the appropriate domain subject to the constraint $\sum_{i} x_{i}=1$ and the removal of cyclic redundancies, cf. Eq. (11). We assumed the asymptotic eigenfunctions to be orthonormal and complete. On the left hand side, we have to evaluate the Hamiltonian matrix elements and multiply the matrix with the column vector of coefficients. In other words, we have to solve an eigenvalue problem for the coefficient vectors. Diagonalizing the full Hamiltonian clearly will yield the coefficients to express the full eigenfunctions as linear combinations of the asymptotic eigenfunctions.

Now, the number of statelets in a state is equal to the order of the group $|\mathcal{G}|=2 r$ ! and grows exponentially. This limits the practical value of the approach. However, it is in some sense the worst case scenario. Often, accidental symmetries arise due to special combinations of excitation numbers $n_{i}$. For instance, the four-parton eigenfunctions, Eq. (4.13) in Ref. [1], can be generated by symmetrizing the statelet $\left|n_{1}, 0, n_{2}\right\rangle$. Most of its statelets will not exhibit a vanishing excitation number, yet the state as a whole is more symmetric than the generic four-parton eigenstate. To wit, it possesses an additional $Z_{2}$ symmetry, ${ }^{13}$ so that the 24 independent statelets can be cast into 12 sinusoidal functions, or into the 6 double-sines of Eq. (4.13) in Ref. [1]. Analogous symmetries seem to exist in all higher partonsectors, e.g., six-parton states $\left|n_{1}, 0, n_{2}, 0, n_{3}\right\rangle$ generating states $^{14}$ of the form Eq. (4.15) in Ref. [1]. All symmetries will reduce the eigenvalue problem further by blockdiagonalizing the Hamiltonian and may give an intuitive understanding of the bosonization process, by which trivial multiparticle states are projected out [9].

So far the emphasis has been on symmetries of the set of excitation numbers. This is natural, since the $\mathcal{S}$ symmetries were introduced exactly for this purpose in Eq. (23). A wave function symmetrized with respect to its excitation numbers is clearly also symmetrized with respect to its arguments, i.e., momentum fractions. What kind of momentum space symmetries do the $N(r) \mathcal{S}$ operators represent? It should be enough to explicitly look at only two, since all others can be derived from them. Namely, $\mathcal{S}_{1}$ and $\mathcal{S}_{2}$ can be considered stereotypical single- and double-neighbor permutations, respectively, since in the latter case $n_{2} \rightarrow n_{2}-n_{1}-n_{3}$ is affected by both of the neighboring excitation numbers. It is clear that the shift in excitation numbers comes from the following shift in momentum fractions

$$
\begin{aligned}
\mathcal{S}_{1} & : x_{1} \rightarrow x_{1}, x_{2} \rightarrow x_{2}-x_{1}, x_{3}=-x_{3}, \ldots, x_{r-1} \\
& \rightarrow-x_{r-1}, x_{r} \rightarrow-x_{r}-x_{1}, \\
\mathcal{S}_{2} & : x_{1} \rightarrow-x_{1}-x_{2}, x_{2} \rightarrow x_{2}, x_{3}=-x_{3}-x_{2}, x_{4} \\
& \rightarrow-x_{4}, \ldots, x_{r} \rightarrow-x_{r} .
\end{aligned}
$$

\footnotetext{
${ }^{13}$ Its identity and $\mathcal{S}_{1}$ statelets fulfill $\mathcal{T} Z|r\rangle=\mathcal{Z}|r\rangle$, whereas for the $\mathcal{S}_{2}$ statelets we have $\mathcal{C}^{2}|r\rangle=\mathcal{I}|r\rangle$, so that half the statelets are redundant.

${ }^{14}$ Note that this works in the massless $\mid$ oeoeo $\rangle$ sector, too.
}

Apparently there is only one fundamental operation in momentum space: all (except one) momentum fractions are inverted, and the fractions next to the invariant one are shifted by the invariant fraction. This shows that the condition that the Hamiltonian be Hermitian, and hence that its eigenfunctions are invariant under $\mathcal{S}$, generates a set of momentum space symmetries that grows exponentially with the parton number. Since these generated symmetries are not obvious, the general method may be useful to determine a full set of symmetries of a given Hamiltonian in other theories.

\section{B. The role of pair production}

With the asymptotic solution Eq. (25) at hand, what can we say about the effect of pair production, i.e., parton number violation? The first observation is that the subsequent parton sectors with the same $\mathcal{T}$ parity have opposite $\mathcal{I}$ parity. In other words, the Hamiltonian is sandwiched between a cosine and a sine wave function. The simplest case is the matrix element between the two and four massless parton $\mathcal{T}$ even sectors, ${ }^{15}$

$$
{ }_{-+}\left\langle r=2 ; 1\left|P_{P V}^{-}\right| r=4 ; 1\right\rangle_{+--},
$$

where

$$
\begin{aligned}
|r=2 ; 1\rangle_{-+}= & \cos \pi x \\
|r=4 ; 1\rangle_{+--}= & \sin \pi\left(x_{1}+2 x_{2}+3 x_{3}\right) \\
& -\sin \pi\left(3 x_{1}+2 x_{2}+x_{3}\right) \\
& +\sin \pi\left(x_{1}-2 x_{2}-x_{3}\right) \\
& +\sin \pi\left(x_{1}+2 x_{2}-x_{3}\right),
\end{aligned}
$$

and the latter wave function has an interesting "disappearance" symmetry under $x_{2} \leftrightarrow x_{4}$. In general, we will have to evaluate a matrix element of the form

$$
{ }_{-+}\left\langle\bar{n}\left|P_{P V}^{-}\right| n, m, l\right\rangle_{+--},
$$

where $\bar{n}$ and $n, m, l$ represent the excitation numbers of the two- and four-parton states, respectively, and $P_{P V}^{-}$is defined in Eqs. (9) and (4). Owing to the definition of Hamiltonian and states, Eqs. (4) and (11), we have to do eight integrations over momenta (one plus three for the states, and four for the Hamiltonian), and have one momentum conserving delta-function, as well as five more appearing when we commute through annihilation operators. So we are left with two integrations over a trigonometric function divided by a quadratic function of the momenta. The structure of the final expression is therefore

\footnotetext{
${ }^{15}$ The subscripts here are the $T I(S)$ quantum numbers, consistent with Table I.
} 


$$
{ }_{-+}\left\langle\bar{n}\left|P_{P V}^{-}\right| n, m, l\right\rangle_{+--} \sim \iint \frac{\sin \pi\left(n^{\prime} x+m^{\prime} y\right)}{(x+y)^{2}} d x d y,
$$

where the integers $n^{\prime}$ and $m^{\prime}$ will depend on all excitation numbers $\bar{n}, n, m, l$ of the two and four-parton states. This integral can be expressed in terms of cosine and sine integrals $(\mathrm{Ci}(x), \mathrm{Si}(x))$, and is not divergent. There will be several terms, and cancellations are possible. Thus, for specific states, or in certain sectors ${ }^{16}$ the matrix element may vanish.

This in turn raises the question whether it is systematically possible to find linear combinations of the asymptotic eigenstates for which the annihilation matrix elements are zero. These would then be states with a definite parton number. In other words, we would have succeeded in summing all pair creation effects, effectively "renormalizing" the theory, i.e., formulating it in terms of effective d.o.f. This program is beyond the scope of the present note, but it seems to be viable-judging from previous work. Namely, in [2,3], it was shown that many of the bound states are very pure in parton number. An open question is whether this behavior is generic or due to the finite discretization used in $[2,3]$.

\section{Implications for the bosonized theory}

$\mathrm{QCD}_{2 A}$ can be bosonized by rewriting the Hamiltonian in terms of current operators $J(-p) \sim \int d q b(q) b(p-q)$ subject to a Kac-Moody algebra, see e.g., Refs. [8,14]. Bosonization is in essence a basis transformation, so the eigenfunctions will change while the eigenvalues, i.e., the bound state masses, remain invariant. Straightforward bosonization generates a nonorthonormal basis, with states consisting of color traces of adjoint current operators acting on a vacuum state. ${ }^{17}$ Numerical approaches [8] produce orthonormal solutions; i.e., the coordinate vectors associated with a chosen basis (whether orthonormal or not) are mutually orthogonal and of unit length. In this sense, a basis of symmetrized harmonics as produced by our "eLCQ" method will be orthonormal.

To get a handle on the bosonized theory, current-number changing operators can be omitted at first [14], and one arrives at an integral equation for the bosonized eigenfunctions $\phi_{r B}$,

$$
\begin{aligned}
& \frac{M^{2}}{g^{2} N} \phi_{r B}\left(x_{1}, \ldots, x_{r}\right) \\
& \quad=-\sum_{i=1}^{r} \int_{-\infty}^{\infty} \frac{\phi_{r B}\left(y, x_{i}+x_{i+1}-y, x_{i+2}, \ldots, x_{i+r-1}\right)}{\left(x_{i}-y\right)^{2}} d y,
\end{aligned}
$$

\footnotetext{
${ }^{16}$ Of course, the $\mathcal{T}$ sector is fixed, but the result can be different in the massive and massless sectors of the theory.

${ }^{17}$ Either the traditional vacuum state or an "adjoint vacuum," represented by a fermionic operator of zero momentum acting on the vacuum.
}

where we have used the subscript $B$ to stress that the partons in the bosonized theory are currents and not fermions. The equation is virtually identical with Eq. (12) save for the nonalternating signs. These signs stemming from anticommuting fermionic operators are clearly absent in the bosonized theory. We should therefore be able to describe the bosonized wave functions with our "eLCQ" ansatz, Eq. (25). We cannot hope for a perfect match, because we cannot decouple sectors of different current number. A consistent asymptotic theory does not exist due to the nature of the Kac-Moody algebra of the current operators. There is an associated problem. Namely, the full theory contains uninteresting nontrivial multiparticle states which interact with the single-particle states of interest at any finite resolution in discretized versions of the theory $[4,12]$. Indeed, only a few single-particle states have hitherto been identified as such. Furthermore, bosonization only works for massless fermions, so there is no massive sector. Hence, our comparison of numerical and algebraic wave functions will be rather limited. Of course, in the bosonized theory, there is a bosonic and also a fermionic sector. In the latter, the basis states are different owing to the elimination of cyclic symmetry due to the existence of a unique fermionic operator (the "adjoint vacuum"). Here, we will focus on the bosonic sector of the bosonized theory. This is a real test of our ansatz, since the symmetrization clearly cannot be the same as in the theory with fermions. The "eLCQ" ansatz (25) for the two-current wave function $\phi_{2 B}$ is

$$
\phi_{2 B}=e^{i n x}+(-1)^{n} e^{-i n x}
$$

(compare to Eq. (B1) which has a minus sign between the terms). Therefore, we "predict" that the massless states with odd $n$ will be sines in the bosonized theory, not cosines as in the fermionic theory. This is consistent with Ref. [14], and also the numerical bosonized eigenfunctions in Fig. 4(b) are consistent with our ansatz. ${ }^{18}$ Note that numerical approaches such as DLCQ use different bases for the fermionic and bosonized theories. The fermionic theory is approximated using antiperiodic boundary conditions (odd half-integer momentum fractions), whereas the bosonized theory uses periodic boundary conditions (integer momentum fractions). Of course, regardless of the basis used, they should be decent approximations to the algebraic wave function.

In sum, we find that the "eLCQ" ansatz is compatible with the known solutions of the theory in a significantly different representation. This hints at a wider applicability of our method. What seems crucial is that the structure of the Hamiltonian be of the form

\footnotetext{
${ }^{18} \mathrm{We} \quad$ used $\quad \phi_{2 B}=\sin \left(\pi x_{1}\right)$ and $\phi_{3 B}=-\frac{1}{20}\left[\cos \left(\pi x_{1}\right)+\right.$ $\left.\cos \left(\pi x_{2}\right)+\cos \left(\pi x_{3}\right)\right]$ to describe the generic features of the two wave functions. Note that the cosines in the higher parton sectors are consistent with our earlier finding that wave functions of same $T$ have opposite $I$ at subsequent $r$.
} 


$$
P^{-} \sim \int \frac{d p}{p^{2}} J_{i j}(-p) J_{j i}(p) .
$$

In other words, the "eLCQ" ansatz is poised to solve the long-range Coulomb-type part of a strongly interacting system. This is akin to the DLCQ ansatz which was shown in Ref. [13] to decouple the center of mass motion of a system from its more interesting physics.

\section{CONCLUSION AND DISCUSSION}

The goal of this paper was to find a complete set of eigenfunctions of $\mathrm{QCD}_{2 A}$. We succeeded in constructing a basis of the asymptotic theory without pair-production, consisting of multidimensional harmonic functions. In order to completely and exhaustively symmetrize the wave functions in the $r$ parton sector, a group of operators of order $2 \mathrm{r}$ ! is necessary and sufficient. This finite group of abstract symmetries is defined by the relations between the generators Eqs. (31)-(33). Paradoxically, this rather complicated arithmetic is based on the simple observation that "boundary conditions" on the wave functions have to be implemented as symmetries in a Hamiltonian approach (which leads to an integral equation for the eigenfunctions). Our finding that the eigenfunctions can be constructed largely algebraically with group theory arguments is corroborated by comparison with numerical solutions of the theory.

At first glance, our method of exhaustively symmetrizing a system quantized on the light-cone ("eLCQ") applies to a specific family of systems, namely theories with adjoint d.o.f. in one spatial dimension with a Coulomb-type longrange interaction. It seems likely, though, that the method presented here is more widely applicable due to its generality. In particular, exhaustive symmetrization deals with momentum as an abstract entity; i.e., it deals with it regardless of its Lorentz structure. There might well be requirements due to the Poincaré group, but this is not the point here. Rather, the fact that we have $r$ momenta requires us to symmetrize on purely abstract grounds, rather as a preconditioning of the wave functions akin to the Slater determinant implementing the antisymmetrization requirement of the Pauli principle.

We presented evidence that "eLCQ" is a useful method at least for theories exhibiting similar integral equations as $\mathrm{QCD}_{2 A}$ - like its bosonized version as discussed in Sec. III C. Other theories to which "eLCQ" can be straightforwardly applied include two-dimensional YangMills theory coupled to adjoint scalars [2] and a theory with adjoint Dirac fermions tackled in [15]. Also, many of the supersymmetric models with adjoint particles (for instance [16]) could be re-evaluated with the present method.

We did not have the space to fully exploit the fact that the asymptotic spectrum of $\mathrm{QCD}_{2 A}$ is now completely mapped out, so there are several opportunities for future projects. For instance, the interaction of asymptotic states and the formation of multiparticle states can be studied. In Sec. III B, we laid down an initial plan how to proceed. And while the exponential rise of terms in the eigenfunctions presents some difficulty, one might be able to improve the precision of numerical solutions enough by using "eLCQ" basis functions to positively identify the single-particle content of the theory. Accidental symmetries described in Sec. III A will help in this regard. Also, the structure of the asymptotic solutions may help to disentangle salient features of this and other theories. For instance, the "topological sector" of the theory, introduced in [4] to explain the appearance of fermion-fermion multiparticle states in the fermionic sectors, might be an artifact of finite group theory. As long as parton number is finite, there is a clear separation of group properties according to their (finite) order. Some combination rules for multiparticle states in terms of $T$ quantum numbers found in [4] might just be the result of such "artificial" group theoretical relations.

\section{ACKNOWLEDGMENTS}

I thank Otterbein University for providing a work environment which made it possible to see this long-term project through to completion. The hospitality of the Ohio State University's Physics Department, where most of this work was completed, is gratefully acknowledged. I would also like to acknowledge the late Hans-Christian Pauli whose work, starting with [13], inspired the method of exhaustive symmetrization developed in this paper.

\section{APPENDIX A: DERIVATION OF THE GROUP MULTIPLICATION TABLE}

To fully understand why there is only one quantum number $S$ associated with the large set $\mathcal{E}$ of $\mathcal{S}$ operators, we work out the details here. We start with the simplest case $r=4$. We need to show that the fully symmetrized wave function $\mathcal{G}|r\rangle$ is an eigenstate to all three $\mathcal{S}$ operators associated with its three excitations numbers $(n, m, l)$. Note that de facto there are only two independent $\mathcal{S}$ operators at $r=4$, which we also have to explain, along with the fact that two thirds - not half — of a state's statelets carry an $S$ sign.

First, we observe that the $4 r$ members of the subgroup $\mathcal{B}$ naturally split into four subsets, namely the cosets of the cyclic subgroup $\langle\mathcal{C}\rangle$ under $\mathcal{T}, \mathcal{I}, \mathcal{T}$, and $\mathcal{I} \mathcal{T}$. But $\mathcal{I}$ is in the center of $\mathcal{G}$, and therefore can be largely ignored. The cyclic structure of the Hamiltonian means that there is a conserved quantity which we might call $\mathcal{T C}$-parity. Odd and even powers of the cyclic group carry different signs in general. Therefore, an even(odd) power has to be mapped under any $\mathcal{S}$ operation onto an even(odd) power of $\mathcal{C}$. When we act with $\mathcal{T}$, then the combined power has to be the same modulo two, e.g., $\mathcal{C}^{\bmod _{2} j} \leftrightarrow \mathcal{T} \mathcal{C}^{\bmod _{2}(j-1)}$. There is a twist. Namely, for states with mixed even and odd excitation 
numbers like $|o e o\rangle$, there is an additional sign, which shifts the relative power under $\mathcal{T}$, so we have $\mathcal{C}^{\bmod _{2} j} \leftrightarrow \mathcal{T} \mathcal{C}^{\bmod _{2} j}$. So the subgroup $\mathcal{B}$ has two subsets even and odd under $\mathcal{T} C$ parity, $\mathcal{B}^{e}$ and $\mathcal{B}^{o}$. We call their members (i.e., combinations like $\left.\mathcal{I}^{i} \mathcal{T}^{t} \mathcal{C}^{c}\right) \mathcal{Z}_{j}^{e}$ and $\mathcal{Z}_{j}^{o}$. At $r=4$ we have the pseudocommutation relations ${ }^{19}$

$$
\begin{aligned}
& \mathcal{S}_{i} \mathcal{Z}_{j}^{e}=\mathcal{Z}_{j}^{e} \mathcal{S}_{i}, \quad \text { (identity preserving) } \\
& \mathcal{S}_{i} \mathcal{Z}_{j}^{e}=\mathcal{Z}_{j}^{e} \mathcal{S}_{\bmod _{2}^{\prime}(i+1)} \quad \text { (identity swapping) }
\end{aligned}
$$

Note that

$$
\mathcal{S}_{i} \mathcal{B}^{e}=\mathcal{B}^{e} \mathcal{S}_{i} \quad \text { and } \quad \mathcal{S}_{i} \mathcal{B}^{o}=\mathcal{B}^{o} \mathcal{S}_{\bmod _{2}^{\prime}(i+1)}
$$

i.e., while the individual operators do not pseudocommute, they remain in the same subset. At $r=4$, the following additional identities hold,

$$
\begin{aligned}
& \mathcal{S}_{1} \mathcal{S}_{2}=T \mathcal{Z}_{k}^{o} \mathcal{S}_{1}=T \mathcal{S}_{2} \mathcal{Z}_{k}^{o}, \\
& \mathcal{S}_{2} \mathcal{S}_{1}=T \mathcal{Z}_{k}^{o} \mathcal{S}_{2}=T \mathcal{S}_{1} \mathcal{Z}_{k}^{o},
\end{aligned}
$$

for some $k$, so that $\mathcal{Z}_{k}^{o} \in \mathcal{B}^{o}$. In fact, $\mathcal{Z}_{k}^{o}=\mathcal{T} C^{2}$. In other words, the product of the two $\mathcal{S}$-operators can be reduced to the leading one, but an odd $\mathcal{Z}$ operator appears as well as an additional $\mathcal{T}$ operator which switches the $T$ sign. We are now ready to calculate the action of $\mathcal{S}_{i}$ on the totally symmetrized state. There are two versions of that state. If focusing on $\mathcal{S}$ symmetry properties, it is natural to write the state in terms of left coset statelets $\mathcal{S} B|r\rangle$

$$
\begin{aligned}
\mathcal{G}|r=4\rangle= & \left(\mathcal{B}^{e}+\mathcal{B}^{o}\right)|r\rangle+S \mathcal{S}_{1}\left(\mathcal{B}^{e}+\mathcal{B}^{o}\right)|r\rangle \\
& +S \mathcal{S}_{2}\left(\mathcal{B}^{e}+\mathcal{B}^{o}\right)|r\rangle .
\end{aligned}
$$

On the other hand, we have to preserve cyclical properties due to the structure of the Hamiltonian, in which case we should use right coset statelets $\mathcal{B S}|r\rangle^{20}$

$$
\begin{aligned}
\mathcal{G}|r=4\rangle= & \left(\mathcal{B}^{e}+\mathcal{B}^{o}\right)|r\rangle+S\left(\mathcal{B}^{e}+\mathcal{B}^{o}\right) \mathcal{S}_{1}|r\rangle \\
& +S\left(\mathcal{B}^{e}+\mathcal{B}^{o}\right) \mathcal{S}_{2}|r\rangle .
\end{aligned}
$$

Both requirements lead to the same constraint on the quantum numbers. In the latter case, we have

$$
\begin{aligned}
\left.\mathcal{S}_{1} \mathcal{G} \mid r=4 ; \text { eee }\right\rangle & =\mathcal{S}_{1}\left(\mathcal{B}^{e}+\mathcal{B}^{o}\right)|r\rangle+S \mathcal{S}_{1}\left(\mathcal{B}^{e}+\mathcal{B}^{o}\right) \mathcal{S}_{1}|r\rangle+S \mathcal{S}_{1}\left(\mathcal{B}^{e}+\mathcal{B}^{o}\right) \mathcal{S}_{2}|r\rangle \\
& =\left(\mathcal{B}^{e} \mathcal{S}_{1}+\mathcal{B}^{o} \mathcal{S}_{2}\right)|r\rangle+S\left(\mathcal{B}^{e}+\mathcal{B}^{o} \mathcal{S}_{2} \mathcal{S}_{1}\right)|r\rangle+S\left(\mathcal{B}^{e} \mathcal{S}_{1} \mathcal{S}_{2}+\mathcal{B}^{o}\right)|r\rangle \\
& =S\left(\mathcal{B}^{e}+\mathcal{B}^{o}\right)|r\rangle+\left(\mathcal{B}^{e}+S T \mathcal{B}^{o}\right) \mathcal{S}_{1}|r\rangle+\left(S T \mathcal{B}^{e}+\mathcal{B}^{o}\right) \mathcal{S}_{1}|r\rangle \\
& =S \mathcal{G} \mid r=4 ; \text { eee }\rangle \quad(\text { if } S T=1),
\end{aligned}
$$

where we used Eq. (A3) in the last step. The calculation goes analogously for $\mathcal{S}_{2}$. We thus proved that a symmetrized state is an eigenstate of $\mathcal{S}_{1}$ (and $\mathcal{S}_{2}$ ) in the $|e e e ; T \pm S \pm\rangle$ sector of the theory. In the mixed sector, the $\mathcal{C}^{j}$ and $\mathcal{T} \mathcal{C}^{j-1}$ terms get an extra sign for odd $j$ due to $(-1)^{n}=(-1)^{l}=-1$. This effectively reverses $T$, therefore the viable sectors are $|o e o ; T \mp S \pm\rangle$.

If we organize in terms of the left cosets we come to the same conclusion. To wit

\footnotetext{
${ }^{19}$ In full detail, they read

$$
\begin{aligned}
& \mathcal{S}_{1} \mathcal{C}=\mathcal{I} \mathcal{T} \mathcal{S}_{2} \quad \mathcal{S}_{1} \mathcal{C}^{2}=\mathcal{I T C S}_{1} \quad \mathcal{S}_{1} \mathcal{C}^{3}=\mathcal{C}^{3} \mathcal{S}_{2} \\
& \mathcal{S}_{1} \mathcal{T}=\mathcal{I C S}_{2} \quad \mathcal{S}_{1} \mathcal{T C}=\mathcal{I C}^{2} \mathcal{S}_{1} \quad \mathcal{S}_{1} \mathcal{T} \mathcal{C}^{2}=\mathcal{T C}^{2} \mathcal{S}_{2} \quad \mathcal{S}_{1} \mathcal{T} \mathcal{C}^{3}=\mathcal{T} \mathcal{C}^{3} \mathcal{S}_{1} \\
& \mathcal{S}_{1} \mathcal{I}=\mathcal{I S}_{1} \quad \mathcal{S}_{1} \mathcal{I C}=\mathcal{T} \mathcal{S}_{2} \quad \mathcal{S}_{1} \mathcal{I C} \mathcal{C}^{2}=\mathcal{T C S}_{1} \quad \mathcal{S}_{1} \mathcal{I} \mathcal{C}^{3}=\mathcal{I C}^{3} \mathcal{S}_{2} \\
& \mathcal{S}_{1} \mathcal{I} \mathcal{T}=\mathcal{C S}_{2} \quad \mathcal{S}_{1} \mathcal{I} \mathcal{T C}=\mathcal{C}^{2} \mathcal{S}_{1} \quad \mathcal{S}_{1} \mathcal{I} \mathcal{T C}^{2}=\mathcal{I T C}^{2} \mathcal{S}_{2} \quad \mathcal{S}_{1} \mathcal{I} \mathcal{T} C^{3}=\mathcal{I T C}^{3} \mathcal{S}_{1} \\
& \mathcal{S}_{2} \mathcal{C}=\mathcal{C S}_{1} \quad \mathcal{S}_{2} \mathcal{C}^{2}=\mathcal{I T} \mathcal{C}^{3} \mathcal{S}_{2} \quad \mathcal{S}_{2} \mathcal{C}^{3}=\mathcal{I} \mathcal{T} \mathcal{S}_{1} \\
& \mathcal{S}_{2} \mathcal{T}=\mathcal{I C}^{3} \mathcal{S}_{1} \quad \mathcal{S}_{2} \mathcal{T C}=\mathcal{T C \mathcal { S } _ { 2 }} \quad \mathcal{S}_{2} \mathcal{T} \mathcal{C}^{2}=\mathcal{T C}^{2} \mathcal{S}_{1} \quad \mathcal{S}_{2} \mathcal{T} \mathcal{C}^{3}=\mathcal{I C}^{2} \mathcal{S}_{2} \\
& \mathcal{S}_{2} \mathcal{I}=\mathcal{I S}_{2} \quad \mathcal{S}_{2} \mathcal{I C}=\mathcal{I C S}_{1} \quad \mathcal{S}_{2} \mathcal{I C}=\mathcal{T C}^{3} \mathcal{S}_{2} \quad \mathcal{S}_{2} \mathcal{I C}^{3}=\mathcal{T} \mathcal{S}_{1} \\
& \mathcal{S}_{2} \mathcal{I} \mathcal{T}=\mathcal{C}^{3} \mathcal{S}_{1} \quad \mathcal{S}_{2} \mathcal{I} \mathcal{T C}=\mathcal{I} \mathcal{T C} \mathcal{S}_{2} \quad \mathcal{S}_{2} \mathcal{I} \mathcal{T} \mathcal{C}^{2}=\mathcal{I T C}^{2} \mathcal{S}_{1} \quad \mathcal{S}_{2} \mathcal{I} \mathcal{T} \mathcal{C}^{3}=\mathcal{C}^{2} \mathcal{S}_{2}
\end{aligned}
$$

\footnotetext{
${ }^{20}$ If this seems inconsistent, recall that the fully symmetrized state is symmetrized both in $\mathcal{B}$ and in $\mathcal{S}$. Hence, though parts of the state will appear to be symmetrized with respect to only one symmetry, the state as a whole has to be symmetric with respect to both operations. In general, this is only possible for certain combinations of quantum numbers. This is the reason why many sectors of the theory do not give rise to proper states.
} 


$$
\begin{aligned}
\mathcal{S}_{1} \mathcal{G} \mid r & =4 ; \text { eee }\rangle=\mathcal{S}_{1}\left(\mathcal{B}^{e}+\mathcal{B}^{o}\right)|r\rangle+S\left(\mathcal{B}^{e}+\mathcal{B}^{o}\right)|r\rangle+S \mathcal{S}_{1} \mathcal{S}_{2}\left(\mathcal{B}^{e}+\mathcal{B}^{o}\right)|r\rangle \\
& =\mathcal{S}_{1}\left(\mathcal{B}^{e}+\mathcal{B}^{o}\right)|r\rangle+S\left(\mathcal{B}^{e}+\mathcal{B}^{o}\right)|r\rangle+S T \mathcal{S}_{2} \mathcal{Z}^{o}\left(\mathcal{B}^{e}+\mathcal{B}^{o}\right)|r\rangle \\
& =S\left[\left(\mathcal{B}^{e}+\mathcal{B}^{o}\right)|r\rangle+S \mathcal{S}_{1}\left(\mathcal{B}^{e}+\mathcal{B}^{o}\right)|r\rangle+T \mathcal{S}_{2}\left(\mathcal{B}^{e}+\mathcal{B}^{o}\right)|r\rangle\right] \\
& =S \mathcal{G} \mid r=4 ; \text { eee }\rangle \quad(\text { if } S=T) .
\end{aligned}
$$

The $I$ quantum number is necessarily the opposite of $T$. The reason is that in the four-parton sector the pseudocommutation relations are such that $\mathcal{T} \mathcal{I}$-parity $(-1)^{t+i}$ is conserved: commuting a (primary) $\mathcal{S}$-operator with a $\mathcal{B}$ operator will yield a different $\mathcal{B}$ operator with equal $t+$ $i$ (modulo two). This essentially links the identity and $\mathcal{T}$ statelets of $\mathcal{S}_{1}$ to the $\mathcal{I} \mathcal{T}$ and $\mathcal{I}$ statelets of $\mathcal{S}_{2}$, respectively. Therefore $T$ and $I$ have to be different, ${ }^{21}$ because otherwise the wave function is identically zero.

The higher parton sectors, $r>4$, are harder to analyze because the pseudocommutation relations are more complicated. In particular, there is no identity preserving relation akin to Eq. (A1). Rather, we have to use Eq. (32). Here $\mathcal{I}$ is decoupled, and $I$-parity thus independently conserved. So we have to come up with an "operator calculus" that allows us to evaluate the $S$ sign of a combination of operators. As it turns out, $r=5$ is not the most general case, but tractable and yielding clues for the generic case $r>5$, so we discuss it here.

The exhaustively symmetrized state in terms of left coset elements can formally be written as

$$
\mathcal{G}|r\rangle=\mathcal{B}|r\rangle+\sum_{j=1}^{N(r)} S_{j} \mathcal{S}_{j} \mathcal{B}|r\rangle=\left(1+\sum_{j=1}^{N(r)} S_{j} \mathcal{S}_{j}\right) \mathcal{B}|r\rangle,
$$

where the $r$ primary operators $\mathcal{S}_{j}$ carry an $S$ sign, so $S_{j}=S$ for $j \leq r$. The nonprimary operators $\mathcal{S}_{j}$ carry an unknown sign $S_{j}= \pm S$. As before, $N(r)=\frac{1}{2}(r-1) !-1=11$, $59,359, \ldots$ The action of a primary operator $\mathcal{S}_{k}$ on an exhaustively symmetrized state is then

$$
\begin{aligned}
\mathcal{S}_{k} \mathcal{G}|r\rangle & =\left(\mathcal{S}_{k}+S+\sum_{\substack{j=1 \\
j \neq k}}^{N(r)} S_{j} \mathcal{S}_{k} \mathcal{S}_{j}\right) \mathcal{B}|r\rangle \\
& =S\left(1+S \mathcal{S}_{k}+S \sum_{\substack{j=j \\
1 \neq k}}^{N(r)} S_{j} \mathcal{S}_{k} \mathcal{S}_{j}\right) \mathcal{B}|r\rangle,
\end{aligned}
$$

where we have used $S_{k}=S$ for primary operators. Due to the group axioms, the secondary operators $\mathcal{S}_{k} \mathcal{S}_{j}$ can be expressed in terms of a single $\mathcal{S}$ operator followed by operators of the $\mathcal{B}$ subgroup, to wit

\footnotetext{
${ }^{21}$ In fact, this yields exactly two sectors, which makes sense in the even $r$ sectors, since here we have two excitation number sectors (even and mixed) for the required four (two massive and massless) sectors. In the odd $r$ sectors, there is only one condition due to the fact that there is no sign due to relative $\mathcal{C}$ operators; at odd $r$ all cyclic permutations enter with a positive sign.
}

$$
\mathcal{S}_{k} \mathcal{S}_{j}=\mathcal{S}_{l} \mathcal{I}^{i} \mathcal{T}^{t} \mathcal{C}^{c}
$$

where the index $l$ and the powers $i, t, c$ can be looked up in a table like Table II. But to reproduce the exhaustively symmetrized state $\mathcal{G}|r\rangle$, we need to have

$$
S S_{j} I^{i} T^{t} C^{c}=S_{l}
$$

since the $\mathcal{B}$ operators can be absorbed into $\mathcal{B}|r\rangle$, whence only the quantum numbers $I, T, C$ re-emerge. This is the condition we can use to identify the $S$ signs of the nonprimary operators.

As an example, consider $r=5$, where we can formally set $C=1$, since cyclic permutations at odd $r$ do not carry signs. Acting with $\mathcal{S}_{1}$, say, on $\mathcal{G}|5\rangle$ yields the operator indices and powers displayed in the $\mathcal{S}_{1}$ column of Table II. Since $I^{i} T^{t}=1$ for all of them, Eq. (A6) simplifies to $S S_{j}=S_{l}$, so that $S_{6}=S_{7}=S_{8}=S_{10}=S_{11}=S^{2}$ and $S_{9}=S$. Note the interplay between $\mathcal{S}_{9}$ and $\mathcal{S}_{10}$ : since $\mathcal{S}_{1} \mathcal{S}_{9} \propto \mathcal{S}_{10}$ and $\mathcal{S}_{1} \mathcal{S}_{10} \propto \mathcal{S}_{9}$, one operator gets an $S$ sign, and the other does not. Which one it is has actually to be decided by acting with another primary operator on the exhaustively symmetrized state or by writing the state in terms of right coset statelets.

The general algorithm is then as follows. Initially, we produce an exhaustive list of operators by acting with primary operators on primary operators until no new operators arise. This leads to a group multiplication table, such as Table II for $r=5$ which can be used to write down consistent expressions for primary operators acting on the generic state (with yet undetermined $S$ signs) in terms of left or right coset statelets. The condition that the emerging state has to be an eigenstate of the primary operators determines the signs, and may lead to a further condition on $I$ as a function of $T$. Using the sign condition Eq. (A6) we start with the $r$ known signs $S_{j}$ of the primary operators to get another $r$ signs $S_{l}$, and iterate the process. The only time this goes bad is when both $S_{j}$ and $S_{l}$ are unknown, as in the case of $\mathcal{S}_{9}$ and $\mathcal{S}_{10}$ at $r=5$. In this case we go on to the next $\mathcal{S}_{k}$. Another constraint arises from the different powers of $I$ and $T$ in the group multiplication table. It will lead to a condition which determines one of these quantum numbers in terms of the other, e.g., $I=T$ at $r=5$. At even $r$ there will be another condition linking (the value of) $S$ and $T$, leading to two viable sectors per (even or mixed) excitation number sector, which is what we need to produce exactly four sectors of bound states at every $r$. 


\section{APPENDIX B: SECTORS, SIGNS, AND SINES}

The assignment of symmetry sectors can be confusing due to several signs and quantum numbers involved. Let's straighten things out by considering the simplest case, $r=2$. Here, we have $\phi_{2}\left(x_{1}, x_{2}\right) \doteq|n\rangle$, so we think of the wave function $\phi_{2}$ depending on two momentum fractions $x_{k}$, as being represented by an abstract vector $|n\rangle$ labeled by one excitation number. The orientation symmetry $\mathcal{T}$ of the Hamiltonian acts on states only via their fermionic operators $b_{i j}\left(-x_{k}\right)$. By flipping color indices, $\mathcal{T}$ effectively reverses the order of the operators and thus the order of momentum fractions. To compensate, also the wave function must be rewritten with the last momentum fraction now being first. Wave functions might be even or odd under this reversal, and the trace of operators might or might not acquire a sign under $\mathcal{T}$. To assign a state to a sector, we have to take into account both behaviors. For example, a two-parton state looks like ${ }^{22}$

$$
\left|\Phi_{2}\right\rangle=\frac{1}{N_{c}} \int_{0}^{\frac{1}{2}} d x \phi_{2}(x, 1-x) \operatorname{Tr}[b(-x) b(1-x)]|0\rangle .
$$

Since

$$
\begin{aligned}
\mathcal{T} b_{i j}(-x) b_{j i}(1-x) & =b_{j i}(-x) b_{i j}(1-x) \\
& =\operatorname{Tr}[b(-x) b(1-x)]
\end{aligned}
$$

\footnotetext{
${ }^{22}$ The integral only runs to $\frac{1}{2}\left(\frac{1}{r}\right.$ in general) since operator products with flipped momentum fractions are redundant under the trace. The relative sign between the two Fock states $\operatorname{Tr}[b(-x) b(1-x)]|0\rangle=-\operatorname{Tr}[b(1-x) b(x)]|0\rangle$ will give rise to a wave function odd under argument reversal here, but not in general.
}

is $\mathcal{T}$ even without the need to reverse the arguments, this state belongs to the $\mathcal{T}$ even sector, even though the wave function $\phi_{2}$ is odd under reversal of its arguments, ${ }^{23}$ cf. Eq. (16). Technically, the wave function is represented as

$$
\begin{aligned}
\left|\phi_{2}\right\rangle & =|n\rangle+T \mathcal{T}|n\rangle+I(\mathcal{I}|n\rangle+T \mathcal{I} \mathcal{T}|n\rangle) \\
& =|n\rangle+T(-1)^{n}|-n\rangle+I|-n\rangle+I T(-1)^{n}|n\rangle,
\end{aligned}
$$

and also as ${ }^{24}$

$$
\left|\phi_{2}\right\rangle=|n\rangle+(-1)^{r+1} \mathcal{C}|n\rangle=|n\rangle-(-1)^{n}|-n\rangle,
$$

where we recalled that $\mathcal{T}|n\rangle=\mathcal{C}|n\rangle=(-1)^{n}|-n\rangle$. So for even $n$ we need $T=I$ and the vanishing of the wave function at $x=0$ is guaranteed by the latter equation which produces a sine wave function with $I=-1$. Clearly, the even $n$ states constitute the massive sector. If $n$ is odd, on the other hand, we get cosines with $I=1$. In both cases, $T=-1$ even though the states are $\mathcal{T}$ even, $\mathcal{T}\left|\phi_{2}\right\rangle=\left|\phi_{2}\right\rangle$. In sum, its quantum number $T$ does not directly give away the $\mathcal{T}$ sector of a state, ${ }^{25}$ but a negative (positive) $I$ quantum number always results in a wave function made of sines(cosines).

\footnotetext{
${ }^{23}$ Of course, we can also flip the $b$ operators and the wavefunction arguments, with the same result.

${ }^{24}$ We are deliberately sloppy here with normalization, which will be taken care of by the Hilbert space integral, Eq. (26).
}

[1] D. Kutasov, Nucl. Phys. B414, 33 (1994).

[2] S. Dalley and I. R. Klebanov, Phys. Rev. D 47, 2517 (1993).

[3] G. Bhanot, K. Demeterfi, and I. R. Klebanov, Phys. Rev. D 48, 4980 (1993).

[4] D. J. Gross, A. Hashimoto, and I. R. Klebanov, Phys. Rev. D 57, 6420 (1998).

[5] J. Boorstein and D. Kutasov, in Proceedings of Les Houches 1997, New non-perturbative methods and quantization on the light cone, (1997), pp. 157-165.

[6] E. Katz, G. M. Tavares, and Y. Xu, J. High Energy Phys. 05 (2014) 143.

[7] G. 't Hooft, Nucl. Phys. B75, 461 (1974).

[8] U. Trittmann, Nucl. Phys. B587, 311 (2000); Phys. Rev. D 66, 025001 (2002).
[9] D. Kutasov and A. Schwimmer, Nucl. Phys. B442, 447 (1995).

[10] J. Gomis, Z. Komargodski, and N. Seiberg, SciPost Phys. 5, 007 (2018).

[11] T. Kanazawa, M. Ünsal, and N. Yamamoto, Phys. Rev. D 96, 034022 (2017).

[12] U. Trittmann, Phys. Rev. D 92, 085021 (2015).

[13] H. C. Pauli, Z. Phys. A 319, 303 (1984).

[14] A. Armoni and J. Sonnenschein, Nucl. Phys. B457, 81 (1995).

[15] R. Gopakumar, A. Hashimoto, I. R. Klebanov, S. Sachdev, and K. Schoutens, Phys. Rev. D 86, 066003 (2012).

[16] J. R. Hiller, M. Harada, S. S. Pinsky, N. Salwen, and U. Trittmann, Phys. Rev. D 71, 085008 (2005); J. R. Hiller, S. S. Pinsky, and U. Trittmann, Phys. Lett. B 541, 396 (2002). 